\title{
Gamma-ray Burst Cosmology
}

\author{
F. Y. Wang ${ }^{\mathrm{a}, \mathrm{b}}$, Z. G. Dai ${ }^{\mathrm{a}, \mathrm{b}}$, E. W. Liang ${ }^{\mathrm{c}}$ \\ ${ }^{a}$ School of Astronomy and Space Science, Nanjing University, Nanjing 210093, China \\ ${ }^{b}$ Modern Astronomy and Astrophysics (Nanjing University), Ministry of Education, Nanjing 210093, China \\ ${ }^{c}$ Department of Physics and GXU-NAOC Center for Astrophysics and Space Sciences, Guangxi University, Nanning 530004, China
}

\begin{abstract}
instract
Gamma-ray bursts (GRBs) are the most luminous electromagnetic explosions in the Universe, which emit up to $8.8 \times 10^{54} \mathrm{erg}$ isotropic equivalent energy in the hard X-ray band. The high luminosity makes them detectable out to the largest distances yet 'explored in the Universe. GRBs, as bright beacons in the deep Universe, would be the ideal tool to probe the properties of high-redshift universe: including the cosmic expansion and dark energy, star formation rate, the reionization epoch and the metal enrichment history of the Universe. In this article, we review the luminosity correlations of GRBs, and implications for constraining the cosmological parameters and dark energy. Observations show that the progenitors of long GRBs are massive stars. So it is 'expected that long GRBs are tracers of star formation rate. We also review the high-redshift star formation rate derived from GRBs, and implications for the cosmic reionization history. The afterglows of GRBs generally have broken power-law spectra, so it is 'possible to extract intergalactic medium (IGM) absorption features. We also present the capability of high-redshift GRBs to probe the pre-galactic metal enrichment and the first stars.
\end{abstract}

'Keywords:

Gamma-ray bursts, cosmology, dark energy, star formation rate, reionization.

\section{Introduction}

Gamma-ray bursts (GRBs) are among the most intriguing phenomena in the Universe (for reviews, see Mészáros 2006; Zhang 2007; Gehrels et al. 2009; Kumar and Zhang 2014). Ac'cording to the duration time $T_{90}$, GRBs are usually classified into two classes: long GRBs $\left(T_{90}>2 \mathrm{~s}\right)$ and short GRBs ' $\left(T_{90}<2 \mathrm{~s}\right)$ Kouveliotou et al. 1993). Long GRBs are thought to arise when a massive star $\left(\geq 25 M_{\odot}\right)$ undergoes core col'lapse, but the progenitors of short GRBs are mergers of double neutron star or a neutron star and a black hole binary Woosley and Bloom 2006. Long GRBs can be made "rela"tive standard candles", using luminosity correlations that have 'been found in prompt and afterglow phases (i.e., Amati et al. 2002; Ghirlanda et al. 2004a; Liang and Zhang 2005). In view 'that the history of the Universe during the so-called "dark age" (from cosmic background radiation at $z \sim 1100$ to the 'epoch when first stars were formed around $z \sim 20$ ) is still poorly known (Barkana and Loeb 2001), GRBs, as bright beacons in the deep Universe, would be the unique tool to illuminate the dark Universe and allow us to unveil the reionization history. GRBs provide ideal probes of the formation rate and environmental impact of stars in the high-redshift universe, including the metal enrichment of the intergalactic medium (IGM). Meanwhile, the infrared (IR) and near-IR afterglows of long GRBs are also expected to be detectable out to very high redshifts (Lamb and Reichart 2000; Ciardi and Loeb 2000; Bromm and Loeb 2002; Wang et al. 2012). The reason is that the cosmological time dilation translates higher redshifts to earlier times in the source frame, at which the afterglow is brighter. The afterglow intensities also decrease with time. So according to the standard theory of GRBs, there is little or no decrease in the flux of GRB afterglows at a given observed time with increasing redshift for a single GRB. Consequently, GRBs can be used as powerful probes of the very high redshift Universe. The prospects of using GRBs as a cosmological tool are exciting:

- The high luminosities of GRBs make them detectable out to high redshifts.

- Gamma-ray photons suffer from no extinction when they propagate towards us. But the optical photons from supernovae will suffer extinction from the interstellar medium (ISM);

- The correlations between GRB spectral properties and energetics have been shown to be powerful tools that "standardize" GRB energetics. So GRBs can be used to constrain cosmological parameters and the nature of dark energy (i.e., Dai et al. 2004; Ghirlanda et al. 2004b; Liang and Zhang 2005; Ghirlanda et al. 2006a);

- The progenitors of GRBs are believed to be stellar mass objects. So the intrinsic luminosity of GRB should not depend on the mass of their host galaxy, which has small mass at high redshifts;

- Long GRBs triggered by the death of massive stars, which have been shown to be associated with supernovae (Stanek et al. 2003; Hjorth et al. 2003), provide a complementary technique for measuring star formation rate. They 
also represent a unique probe of the initial mass function and the star formation of massive stars at very high redshifts. GRBs offer the exciting opportunity to detect the Population III (Pop III) stars;

- GRB afterglows have smooth continuum spectra that allow one to extract the IGM absorption features. The metal absorption lines in the GRB spectra make GRBs powerful sources to study the metal enrichment history;

- The clean red damping wings of GRBs make them ideal tools to study the reionization of IGM and ISM properties of their hosts.

First, GRBs can serves as the complementary tools to measure dark energy and cosmic expansion. Type Ia supernovae (SNe Ia) are now treated as ideal "standard candles" for purposes of Hubble diagram. In 1998, two teams studying distant $\mathrm{SNe}$ Ia discovered independent evidence that the expansion of the Universe is speeding up (Riess et al. 1998; Perlmutter et al. 1999), which is attributed to the mysterious component — dark energy. The accelerated expansion of the universe has also been confirmed by several independent observations including those of the cosmic microwave background (CMB) (Spergel et al. 2003), the baryonic acoustic oscillations (BAO) (Eisenstein et al. 2005), X-ray gas mass fraction in galaxy clusters (Allen et al. 2004), and Hubble parameters (Jimenez et al. 2003; Wang and Wang 2014a). The standard $\Lambda$ CDM model fits the observational data well, but other dark energy models could not be ruled out because of the precision of current data. The latest observations confirm that about $70 \%$ of the energy density of the present Universe consists of dark energy. The direct evidence for the current acceleration of the universe is related to the observation of luminosity distances of high redshift SNe Ia. The SNe Ia can be observed when accreting white dwarf stars exceed the mass of the Chandrasekhar limit and explode. Thus they can be treated as an ideal standard candle. But hitherto the highest redshift of SNe Ia is 1.914 (Jones et al. 2013). GRBs are promising tools to study the cosmic expansion at higher redshift, filling the gap between SNe Ia and CMB (Dai et al. 2004; Ghirlanda et al. 2004b; Liang and Zhang 2005; Ghirlanda et al. 2006a; Wang and Dai 2006a; Wang et al. 2007; Schaefer 2007; Capozziello et al. 2012; Wang 2012). Similarly to SNe Ia, it has been proposed to use correlations of GRBs between various properties of the prompt emission and of the afterglow emission to standardize GRB energetics (i,e., Ghirlanda et al. 2004a; Xu et al. 2005; Liang and Zhang 2006; Firmani et al. 2005; Amati 2006; Amati et al. 2008; Liang et al. 2008, 2010).

The high-redshift $(z>6)$ star formation history (SFH) is important in many fields in astrophysics. Direct star formation rate (SFR) measurement beyond the reach of present instruments, particularly at the low part of the galaxy luminosity function. Long GRBs triggered by the death of massive stars, provide a complementary technique for measuring the SFR (Totani 1997; Wijers et al. 1998; Bromm and Loeb 2002). Because the lifetimes of massive stars are short, the SFR can be treated as their death rate. Recent Swift ob- servation shows that GRBs are not tracing the SFR exactly (Daigne et al. 2006; Le and Dermer 2007; Yüksel and Kistler 2007; Salvaterra and Chincarini 2007; Guetta and Piran 2007; Kistler et al. 2008; Campisi et al. 2010; Salvaterra et al. 2012).

The SFR revealed by the high-redshift GRBs seems to be much higher than that obtained from high-redshift galaxy surveys (Kistler et al. 2008, 2009; Wang and Dai 2009). An enhancement about $(1+z)^{\delta}(\delta \sim 0.5-1.5)$ in the observed rate of GRBs compared to SFR has been found (Kistler et al. 2008; Robertson and Ellis 2012; Wang 2013). In order to explain this discrepancy, many models have been proposed, including cosmic metallicity evolution ( $\mathrm{Li}$ 2008; Oin et al. 2010), superconducting cosmic strings (Cheng et al. 2010), evolving star initial mass function Wang and Dai 2011a; Xu and Wei 2008), evolution of the luminosity function break of GRBs Virgili et al. 2011; Yu et al. 2012). But some other studies claimed that there is no discrepancy between GRB rate and SFR (Elliott et al. 2012; Hao and Yuan 2013). From host galaxy observations, long GRBs prefer to form in a low-metallicity environment (Le Floc'h et al. 2003; Stanek et al. 2006; Levesque 2014), which is also required by theoretical prediction. The mass loss of stars through winds is proportional to the metallicity, so low-metallicity can reduce the loss of angular momentum. Some studies have argued that GRB progenitors must have a low metallicity (Woosley and Heger 2006; Mészáros 2006; Langer and Norman 2006). Observations also show differences in the population of GRB host galaxies compared to expectations for an unbiased star-formation tracer (Tanvir et al. 2004; Fruchter et al. 2006; Svensson et al. 2010).

The metal enrichment history has several important consequences for structure formation (Madau et al. 2001; Karlsson et al. 2012). An early phase of metal injection may qualitatively change the character of star formation, from a high-mass (Pop III) mode to a normal, low-mass dominated (Pop I/II) one, if the enrichment exceeds a 'critical metallicity' of $Z_{\text {crit }} \sim 10^{-4} Z_{\odot}$ (Bromm et al. 2001; Schneider et al. 2002, 2006). This mode transition has crucial implications, e.g., for the expected redshift distribution of GRBs (Bromm and Loeb 2006; Campisi et al. 2011; de Souza et al. 2011), for the cosmic reionization (Cen 2003; Wyithe and Loeb 2003; Furlanetto and Loeb 2005), and for the chemical abundance of low-metallicity stars (Oian and Wasserburg 2001; Frebel et al. 2007, 2009; Tumlinson 2010). Absorption lines on the spectra of bright background sources, such as GRBs or quasars, are main sources of information about the chemical properties of high-redshift Universe (Oh 2002; Furlanetto and Loeb 2003; Oppenheimer et al. 2009). These lines are due to absorption by metals in low-ionization stages which arise in the higher column-density gas associated with damped Ly $\alpha$ absorbers (DLAs). GRBs as bright sources have a number of advantages compared to traditional lighthouses such as quasars (Bromm and Loeb 2012). Their number density drops much less precipitously than quasars at $z>6$ (Fan et al. 2006), together with the power-law character of their spectra, renders them ideal probes of the early IGM. Quasars show strong spectral features, such as broad emission lines or the so-called "blue bump" that complicate the extraction of IGM 
absorption features. The analysis of the spectrum of distant GRB 050904 has offered a wealth of detailed insight into the physical conditions of the host galaxy at $z \simeq 6.3$ (Totani et al. 2006). Salvaterra et al. (2009) claimed that they identified two absorption lines (Si IV and Fe II) in the spectrum of GRB 090423, the most distant spectroscopically confirmed burst at $z=8.2$ (Salvaterra et al. 2009; Tanvir et al. 2009).

Besides the above fields, high-redshift GRBs also may be useful tools to study dark matter and primordial nonGaussianities. Because the matter power spectrum is dependent on the mass of dark matter, i.e., cold, warm and hot, which can result in different structure formation. So the number counts of high-redshift GRBs can set strong lower limits on the dark matter mass (Mesinger et al. 2005; de Souza et al. 2013). The rate of high-redshift GRBs is also dependent on the amount of primordial non-Gaussianity in the density field (Maio et al. 2012).

In this work, we review the cosmological implications of GRBs. Then in the following sections the gamma-ray bursts cosmology is reviewed: The second section is dedicated to luminosity correlations and cosmological constraints from GRBs. Section 3 discusses the capability of GRBs to reveal the highredshift SFR and cosmic reionization. In section 4, the capability of GRBs to probe the metal enrichment history is discussed. The last section provides a summary and future prospect.

\section{GRBs as standard candles to probe dark energy}

The best way to measure properties of the dark energy is to measure the expansion history of our universe, i.e., the redshiftdistance relation. To this end, WFIRST has been proposed to determine the distances of 1000 SNe Ia with exquisite accuracy. To explore the properties of dark energy, the best method is to measure it over a wide range of redshifts, but $\mathrm{SNe}$ Ia can only be detected at low redshifts, i.e., $z<2.0$. GRBs can extend the Hubble diagram to high redshifts. So many attempts have been performed to standardize GRBs. Frail et al. (2001) found that the collimated energetics of GRBs clustered around $5 \times 10^{50}$ erg, which was confirmed by Bloom et al. (2003). Observations also require that the GRB emission is only in a small angle (Waxman et al. 1998; Fruchter et al. 1999). The collimated GRB model predicts that the appearance of an achromatic break in the afterglow light curve (Rhoads 1997; Sari 1999). This break is important to standardize the energetic of GRBs.

Similar as SNe Ia, the luminosity correlations are required to probe dark energy using GRBs. In this section, we first review luminosity correlations of GRBs. Then the progress on dark energy revealed by GRBs is discussed. Some reviews have discussed this topic (i.e., Ghirlanda et al. 2006a; Dai and Wang 2007; Capozziello et al. 2012; Amati and Della Valle 2013).

\subsection{The luminosity correlations of GRBs}

The luminosity correlations are connections between parameters of the light curves and/or spectra with the GRB luminosity or energy. The isotropic luminosity can be calculated as

$$
L=4 \pi d_{L}^{2} P_{\text {bolo }}
$$

and the total collimation-corrected energy is

$$
E_{\gamma}=E_{\text {iso }} F_{\text {beam }}=4 \pi d_{L}^{2} S_{\text {bolo }} F_{\text {beam }}(1+z)^{-1} .
$$

Here, $P_{\text {bolo }}$ and $S_{\text {bolo }}$ are the bolometric peak flux and fluence, respectively, while $F_{\text {beam }}=1-\cos \theta_{\text {jet }}$ is the beaming factor with jet opening angle $\theta_{\text {jet }}$. The peak fluxes and fluences are given over a wide variety of observed bandpasses, and with the wide range of redshifts which correspond to different range of energy bands in the rest frame of GRB. So the K-correction is important (Bloom et al. 2001). $P_{\text {bolo }}$ and $S_{\text {bolo }}$ are computed from the differential energy spectrum $\Phi(E)$ as follows:

$$
\begin{gathered}
P_{\text {bolo }}=P \times \frac{\int_{1 /(1+z)}^{10^{4} /(1+z)} E \Phi(E) d E}{\int_{E_{\min }}^{E_{\max }} \Phi(E) d E}, \\
S_{\text {bolo }}=S \times \frac{\int_{1 /(1+z)}^{10^{4} /(1+z)} E \Phi(E) d E}{\int_{E_{\min }}^{E_{\max }} E \Phi(E) d E},
\end{gathered}
$$

with $P$ and $S$ being the observed peak energy and fluence in units of photons $/ \mathrm{cm}^{2} / \mathrm{s}$ and $\mathrm{erg} / \mathrm{cm}^{2}$, respectively, and $\left(E_{\min }, E_{\max }\right)$ the detection thresholds of the observing instrument. In general, the differential energy spectrum is modeled using a broken power - law (Band et al. 1993),

$$
\Phi(E)= \begin{cases}A E^{\alpha} \mathrm{e}^{-(2+\alpha) E / E_{\text {peak }}} & E \leq \frac{\alpha-\beta}{2+\alpha} E_{\text {peak }} \\ B E^{\beta} & \text { otherwise }\end{cases}
$$

where $\alpha$ is the power-law index for photon energies below the break and $\beta$ is the power-law index for photon energies above the break. Some differential energy spectra of GRBs also show power-law spectra plus an exponential cutoff. The luminosity distance $d_{L}$ can be expressed as

$$
d_{L}(z)=(1+z) \frac{c}{H_{0}} \int_{0}^{z} \frac{d z^{\prime}}{E\left(z^{\prime}\right)},
$$

where $E^{2}(z)=\Omega_{\mathrm{M}}(1+z)^{3}+\Omega_{\mathrm{x}} f_{\mathrm{x}}(z)$ and $f_{\mathrm{X}}(z)$ is given by

$$
f_{\mathrm{x}}(z)=\exp \left[3 \int_{0}^{z} \frac{1+w(\tilde{z})}{1+\tilde{z}} \mathrm{~d} \tilde{z}\right]
$$

where $w(z)$ is the equation of state (EOS) of dark energy. For $\Lambda \mathrm{CDM}, \mathrm{Eq} .6$ reduces to

$$
d_{L}(z)=(1+z) \frac{c}{H_{0}} \int_{0}^{z} \frac{d z^{\prime}}{\left.\Omega_{M}\left(1+z^{\prime}\right)^{3}\right)+\Omega_{\Lambda}} .
$$

The promising luminosity correlations of GRBs are as follows:

- $L_{\text {iso }}-\tau_{\text {lag }}$ correlation. The luminosity-time lag correlation was first discovered by Norris et al. (2000) based on six GRBs observed by BATSE with optical redshifts (also see Schaefer et al. 2001), which was confirmed by GRBs observed by Swift (Gehrels et al.2006) including the peculiar long GRB 060218 (Liang and Zhang 2006). But this correlation is challenged by recent study (Bernardini et al. 


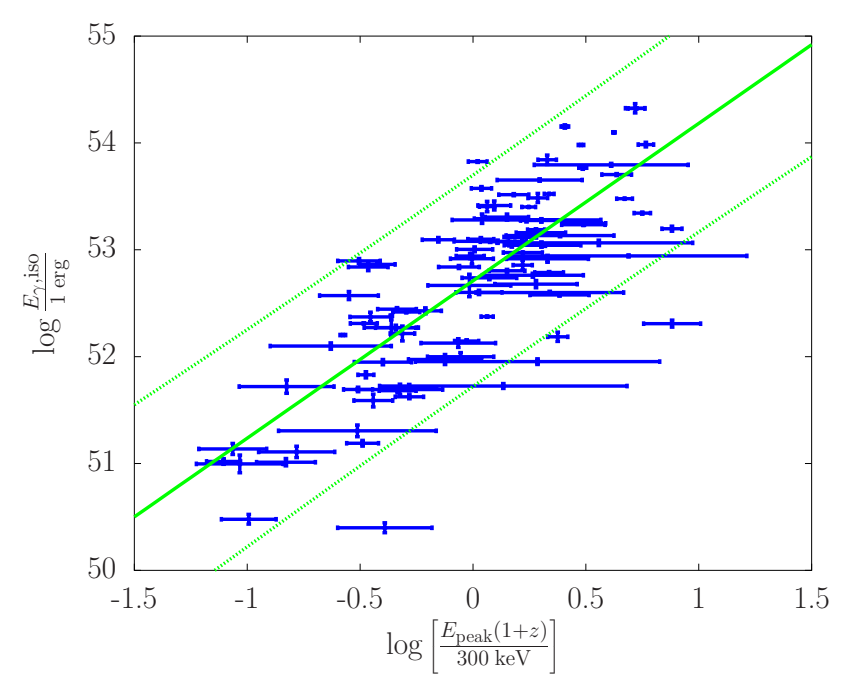

Figure 1: The Amati correlation of 101 GRBs. Solid line is the best fit, and dashed lines represent the $1 \sigma$ dispersion. (Adapted from Figure 1 in Wang et al. (2011).)

2015). This correlation shows that more luminous bursts are also characterized by shorter time lags, i.e., $L_{\text {iso }} \propto$ $\tau_{\text {lag }}^{-1.25}$ (Norris et al. 2000). This correlation has been used as a redshift indicator to estimate $z$ for GRBs (Band et al. 2004), and to constrain cosmological parameters and dark energy (Schaefer 2007; Wang et al. 2007). The interpretation of this correlation is various, including viewing angle of the collimated jet (Ioka and Nakamura 2001) or radiative cooling effect (Crider et al. 1999).

- $L_{\text {iso }}-V$ correlation. Fenimore and Ramirez-Ruiz (2000) found that the time variability is correlated with the luminosity of GRBs, which indicates that more luminous bursts have more variable light curves. Later, this correlation is confirmed with more GRBs by Reichart et al. (2001) and Schaefer et al. (2001). But the intrinsic scatter of $L_{\text {iso }}-V$ is very large (Guidorzi et al. 2006; Wang et al. 2011), and the index is still under debate ( $\mathrm{Li}$ and Paczyński 2006). The variability $V$ is different for various instruments. The origin of the $L_{\text {iso }}-V$ correlation may be based on the screening effect of the photosphere (Kobayashi et al. 2002; Mészáros et al. 2002).

- Amati correlation. Amati et al (2002) found that the isotropic energy $E_{\mathrm{iso}}$ is correlated with the rest-frame peak energy of the prompt spectrum, i.e., $E_{\text {peak }} \propto E_{\text {iso }}^{0.52}$. Subsequent observations with various detectors (such as Swift and Fermi) confirmed this correlation (Amati et al. 2009; Ghirlanda et al. 2010; Sakamoto et al. 2011; Wang et al. 2011). Moreover, it was found that the Amati correlation also holds within individual GRBs using time-resolved spectra, and the slopes are consistent with the correlation from time-integrated spectra (Ghirlanda et al. 2010; Frontera et al. 2012). The possible interpretations of Amati correlation include the synchrotron mechanism in relativistic shocks (Zhang and Mészáros 2002) and emis- sion from off-axis relativistic jets (Yamazaki et al. 2004; Eichler and Levinson 2004). Figure 1 shows the Amati correlation including $101 \mathrm{GRBs}$. The intrinsic scatter is 0.62 .

- Yonetoku correlation. The correlation $L_{\text {iso }} \propto E_{\text {neak }}^{2}$ was found with a sample of 16 GRBs (Yonetoku et al. 2004; Wei and Gao 2003). This correlation was confirmed by Liang et al. (2004). Similar as Amati correlation, $E_{\text {peak }}-L_{\text {iso }}$ also holds within individual GRBs using time-resolved spectra (Ghirlanda et al. 2010). The possible origin of this correlation is similar as that of Amati correlation. Figure 2 shows the Yonetoku correlation. The intrinsic scatter of this correlation is 0.62 (Wang et al.2011).

- Ghirlanda correlation. A tight correlation between spectral peak energy $E_{\text {peak }}$ and collimated energy $E_{\gamma}$ was discovered by Ghirlanda et al. (2004a) using 15 GRBs. The intrinsic scatter is up to 0.1 , so this correlation is a promising tool to constrain dark energy (Dai et al. 2004; Ghirlanda et al. 2004b). By considering the wind circumburst density, Nava et al. (2006) found this correlation also holds, and the intrinsic scatter is even smaller. One of the major challenge for this correlation is that most of GRBs observed by Swift do not show achromatic breaks in the afterglow light curve (Willingale et al. 2007). So the break time is very hard to determine. This correlation can be understood within the annular jet model (Eichler and Levinson 2006) and photosphere model (Thompson 2006). The latest Ghirlanda correlation is shown in Figure 3

- $L_{\text {iso }}-E_{\text {peak }}-T_{0.45}$ correlation. Firmani et al. (2006) found a tight correlation $L_{\text {iso }} \propto E_{\text {peak }}^{1.62} T_{0.45}^{-0.49}$ using parameters in prompt emission only. $T_{0.45}$ represents the "high signal" timescale.

- Liang-Zhang correlation. Without imposing any theoretical model, Liang and Zhang (2005) found an empirical correlation among the isotropic energy of the prompt gamma-ray emission $E_{\text {iso }}$, the rest-frame peak energy $E_{\text {peak }}$, the rest-frame break time in the optical band $t_{\text {break }}$ using 15 bursts. The correlation reads $E_{\text {peak }} \propto E_{\text {iso }}^{0.52} t_{\text {break }}^{0.64}$. If we take the optical break time as the jet break time, this correlation is similar to Ghirlanda correlation. The intrinsic scatter of this correlation is also very small, so it could be used to constrain dark energy (Liang and Zhang 2005; Wang and Dai 2006a; Wei et al. 2013). The Liang-Zhang correlation is shown in Figure 4.

- $L_{X}-T_{a}$ correlation. Dainotti et al. (2008) discovered a tight correlation between X-ray luminosity $L_{X}$ and $T_{a}$, where $T_{a}$ is the time at which the X-ray light curve establishes a afterglow power-law decay Willingale et al. 2007). The intrinsic scatter of this correlation is about 0.33 . By adding a third parameter isotropic energy $E_{\text {iso }}$, $\mathrm{Xu}$ and Huang (2012) found a new correlation, i.e., $L_{X} \propto$ $T_{a}^{-0.87} E_{\mathrm{iso}}^{0.88}$. 


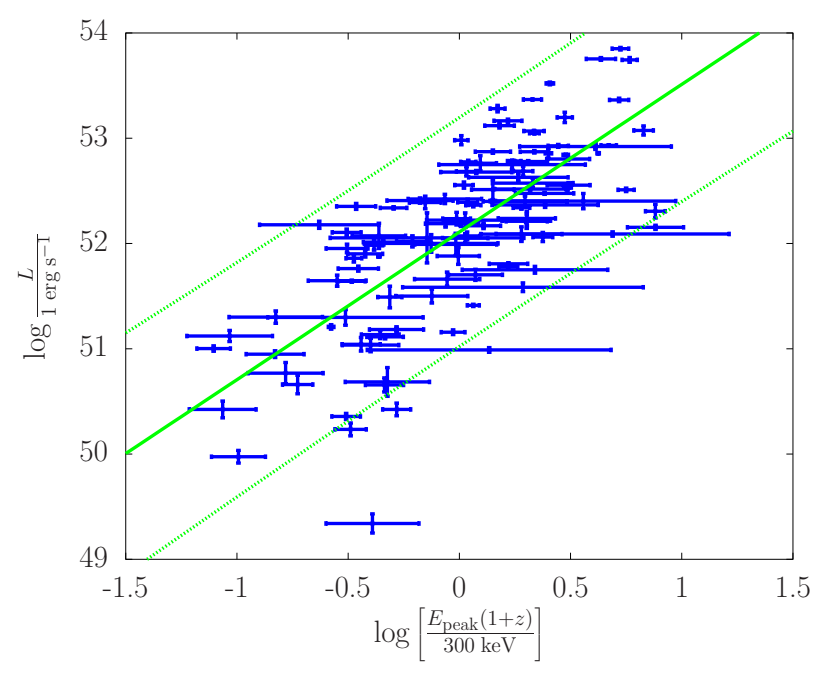

Figure 2: The Yonetoku correlation. Solid line is the best fit, and dashed lines represent the $1 \sigma$ dispersion. (Adapted from Figure 1 in Wang et al. (2011).)

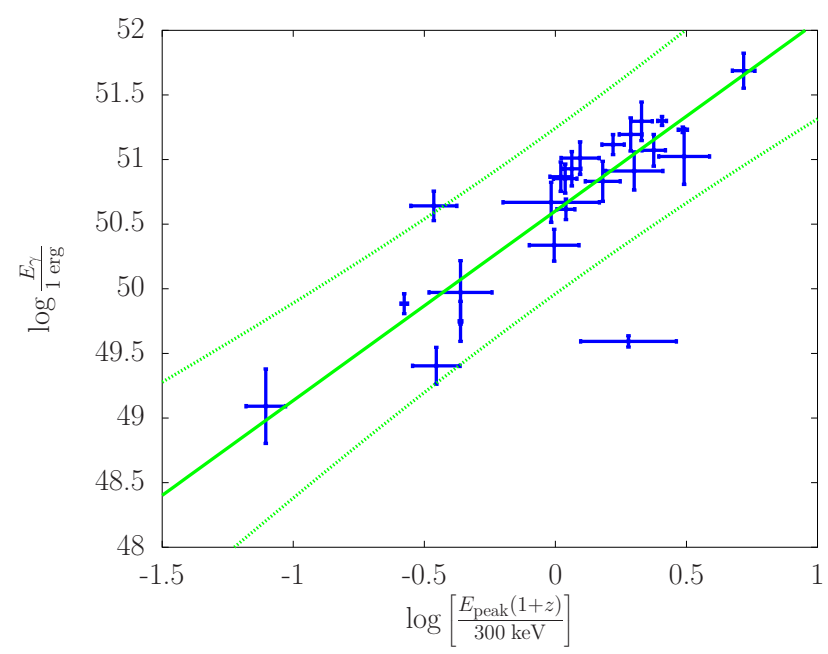

Figure 3: The Ghirlanda correlation. Solid line is the best fit, and dashed lines represent the $1 \sigma$ dispersion. (Adapted from Figure 1 in Wang et al. (2011).)

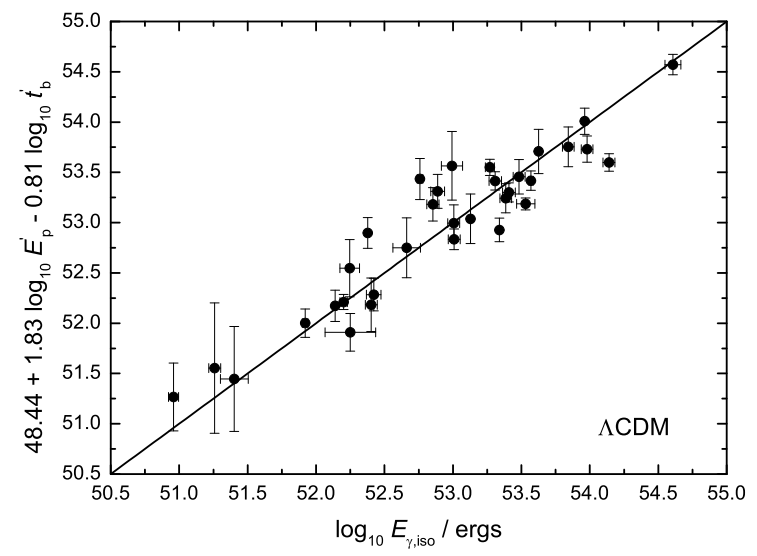

Figure 4: The Liang-Zhang correlation of 33 GRBs in the $\Lambda$ CDM model. (Adapted from Figure 1 in Wei et al. (2013).)
Due to GRBs cover large redshift range, whether the correlations evolve with the redshift should be discussed. It's found that the slope of Amati correlation may vary with redshift significantly using a small sample of GRBs (Li 2007). Basilakos and Perivolaropoulos (2008) found no statistically significant evidence for redshift dependence of slopes in five correlations using 69 GRBs compiled by Schaefer (2007). Wang et al. (2011) enlarge the GRB sample and test six GRB correlations. There is no statistically significant evidence for the evolution of the luminosity correlations with redshift is found. The slopes of correlations versus redshift are all consistent with zero at the $2 \sigma$ confidence level.

The instrumental selection effects may affect the observed luminosity correlations. Some outliers to these correlations have been discovered (Soderberg et al. 2004; Vaughan et al. 2006; Campana et al. 2007; Rizzuto et al. 2007; Urata et al. 2009). Nakar and Piran (2005) considered the different samples of GRBs detected by BATSE. They found that a large fraction of GRBs were inconsistent with the Amati correlation by assuming all possible redshifts. Band and Preece (2005) also found that about 88 and 1.6 per cent of their BATSE GRBs were outliers to the Amati and Ghirlanda correlations, respectively. Butler et al. (2007) claimed that the Amati correlation exists, but it may be due to the selection effect. Using a large sample of GRBs with pseudo-redshifts determined by the $L_{\text {iso }}-\tau_{\text {lag }}$ correlation, Ghirlanda et al. (2005) argued that the Amati correlation really existed by taking into account the intrinsic scatter. By considering the triggering threshold limits for several GRB detectors, Nava et al. (2008) and Ghirlanda et al. (2008) claimed that only $6 \%$ of BATSE long GRBs are inconsistent with Amati correlation, while on outliers to the Ghirlanda correlation are found. By simulating the BATSE Large Area Detectors triggering thresholds, Shahmoradi and Nemiroff (2011) found that the Amati and Ghirlanda correlations are statistically real but strongly affected by the thresholds of GRB detectors. Ghirlanda et al. (2012) studied the selection effect on the Yonetoku correlation using Monte Carlo simulations, and found this correlation must be physical. Dainotti et al. (2014) proposed a general method to check the selection effects for GRB correlations and found the $L_{x}-T_{a}$ correlation is not generated by the biases. Interestingly, using the time-resolved spectra, similar correlations were found in individual bursts (Firmani et al. 2009; Ghirlanda et al. 2010; 2011). This strongly supports that the correlations are physical.

\subsection{Constraints on dark energy and cosmological parameters}

The most common method to constrain dark energy is through its influence on the expansion history of the universe, which can be extracted from the luminosity distance $d_{L}(z)$ and the angular diameter distance $d_{A}(z)$. In addition, the weak gravitational lensing, growth of large-scale structure, and redshift space distortion can also provide useful constraints on dark energy. Theoretical models can be tested using the $\chi^{2}$ statistic. The typical way to probe dark energy from standard candles is as follows. With luminosity distance $d_{L}$ in units of mega- 
parsecs, the theoretically predicted distance modulus is

$$
\mu=5 \log \left(d_{L}\right)+25 .
$$

The likelihood functions for the cosmological parameters can be determined from $\chi^{2}$ statistics,

$$
\chi^{2}\left(\Omega_{M}, \Omega_{D E}\right)=\sum_{i=1}^{N} \frac{\left[\mu_{i}\left(z_{i}, H_{0}, \Omega_{M}, \Omega_{D E}\right)-\mu_{0, i}\right]^{2}}{\sigma_{\mu_{0, i}}^{2}}
$$

where $\mu_{0, i}$ is the observed distance modulus, and $\sigma_{\mu_{0, i}}$ is the standard deviation in the individual distance modulus. The confidence regions in the $\Omega_{M}-\Omega_{D E}$ plane can be found through marginalizing the likelihood functions over $H_{0}$ (i.e., integrating the probability density $p \propto \exp \left(-\chi^{2} / 2\right)$ for all values of $\left.H_{0}\right)$.

A lot of effort had been made to constrain cosmological parameters using GRBs since their cosmological origin was confirmed. Schaefer (2003) obtained the first GRB Hubble diagram based on $L_{\text {iso }}-V$ correlation, and found the mass density $\Omega_{M}<0.35$ at the $1 \sigma$ confidence level. After Ghirlanda et al. (2004a) found the Ghirlanda correlation, Dai et al. (2004) first used this correlation with 12 bursts and found the mass density $\Omega_{M}=0.35 \pm 0.15$ at the $1 \sigma$ confident level for a flat universe by assuming that some physical explanation comes into existence. Ghirlanda et al. (2004b) using 14 GRBs and SNe Ia obtained $\Omega_{\mathrm{M}}=0.37 \pm 0.10$ and $\Omega_{\Lambda}=0.87 \pm 0.23$. Assuming a flat universe, the cosmological parameters were constrained to be $\Omega_{\mathrm{M}}=0.29 \pm 0.04$ and $\Omega_{\Lambda}=0.71 \pm 0.05$ (Ghirlanda et al. 2004b). For a flat universe, Firmani et al. (2005) found $\Omega_{M}=0.28 \pm 0.03$ and $z_{T}=0.73 \pm 0.09$ for the combined GRB and SNe Ia sample, where $z_{T}$ is the transition redshift, at which the universe starts accelerating expansion. In the dark energy model of $w_{z}=w_{0}$, they found $\Omega_{M}=0.44$ and $w_{0}=-1.68$ with $z_{T}=0.40$ for the combined GRB and SNe Ia sample. Xu et al. (2005) obtained $\Omega_{M}=0.15_{-0.13}^{+0.45}(1 \sigma)$ using 17 GRBs. Friedman \& Bloom (2005) discussed several possible sources of systematic errors in using GRBs as standard candles. Using the $E_{\text {peak }}-E_{\text {iso }}-t_{\text {break }}$ correlation, Liang and Zhang (2005) found the $1 \sigma$ constraints are $0.13<\Omega_{\mathrm{M}}<0.49$ and $0.50<\Omega_{\Lambda}<0.85$ for a flat universe. They also found the transition redshift is $0.78_{-0.23}^{+0.32}$. Wang and Dai (2006a) using the Liang-Zhang correlation to investigate the transition redshifts in different dark energy models via GRBs and SNe Ia, see also Wang and Dai (2006b). Di Girolamo et al. (2005) simulated different samples of gamma-ray bursts and found that $\Omega_{M}$ could be determined with accuracy $\sim 7 \%$ with data from 300 GRBs. Meanwhile, many works have been done on this field, such as Mörtsell and Sollerman (2005), Bertolami and Silva (2006), Firmani et al. (2006), Hooper and Dodelson (2007), Li et al. (2008a), Mosquera Cuesta et al. (2008), Basilakos and Perivolaropoulos (2008), Wang (2008), Wang et al. (2009b), Yu et al. (2009), Cardone et al. (2009), Oi and Lu (2010), Cardone et al. (2011), Demianski et al. (2011), Demianski and Piedipalumbo (2011), and Pan et al. (2013). More recently, theoretical arguments and observational evidence both suggested that a small fraction of fast radio bursts (FRBs) may be associated with GRBs (Bannister et al.
2012; Thornton et al. 2013; Zhang 2014). So the dispersion measure from FRBs and redshifts from GRBs makes these systems a plausible tool to study cosmological parameters (Deng and Zhang 2014; Zhou et al. 2014; Gao et al. 2014).

Unfortunately, because of lack of low- $z$ GRBs, the luminosity correlations has been obtained only from moderate- $z$ GRBs. So this correlation is cosmology-dependent, i.e., the isotropic energy $E_{\text {iso }}$, collimation-corrected energy $E_{\gamma}$, and luminosity $L_{\text {iso }}$ are as functions of luminosity distance $d_{L}$, which is dependent on cosmology model. This is the so-called "circularity problem" of GRBs. In the following, we present different methods to overcome this problem.

The first method is fitting the cosmological parameters and luminosity correlation simultaneously. Ghirlanda et al. (2004b) first used this method to overcome the "circularity problem". Schaefer (2007) used 69 GRBs and five correlations to constrain cosmological parameters. Wang et al. (2007) also used 69 GRBs and other cosmological probes to constrain cosmological parameters. They make simultaneous uses of five luminosity indicators, which are correlations of $L_{\text {iso }}-\tau_{\text {lag }}, L_{\text {iso }}-V$, $E_{\text {peak }}-L_{\text {iso }}, E_{\text {peak }}-E_{\gamma}$, and $\tau_{\mathrm{RT}}-L_{\text {iso }}$. After obtaining the distance modulus of each burst using one of these correlations, the real distance modulus can be calculated,

$$
\mu_{\mathrm{fit}}=\left(\sum_{i} \mu_{i} / \sigma_{\mu_{i}}^{2}\right) /\left(\sum_{i} \sigma_{\mu_{i}}^{-2}\right)
$$

where the summation runs from $1-5$ over the correlations with available data, $\mu_{i}$ is the best estimated distance modulus from the $i$-th relation, and $\sigma_{\mu_{i}}$ is the corresponding uncertainty. The uncertainty of the distance modulus for each burst is

$$
\sigma_{\mu_{\mathrm{fit}}}=\left(\sum_{i} \sigma_{\mu_{i}}^{-2}\right)^{-1 / 2}
$$

Because each correlation with its limitations and possible biases, simultaneous using may affect the cosmological indicators. But in order to enlarge the sample, some attempts have been performed. When calculating constraints on cosmological parameters and dark energy, the normalizations and slopes of the five correlations are marginalized. The marginalization method is to integrate over some parameters for all of its possible values. The $\chi^{2}$ value is

$$
\chi_{\mathrm{GRB}}^{2}=\sum_{i=1}^{N} \frac{\left[\mu_{i}\left(z_{i}, H_{0}, \Omega_{M}, \Omega_{D E}\right)-\mu_{\mathrm{fit}, i}\right]^{2}}{\sigma_{\mu_{\mathrm{ft}, i}}^{2}},
$$

where $\mu_{\mathrm{fit}, i}$ and $\sigma_{\mu_{\mathrm{fit}, i}}$ are the fitted distance modulus and its error. In addition to GRBs, SNe Ia, CMB, BAO, X-ray gas mass fraction in galaxy clusters and growth rate data are also ideal cosmological probes. CMB is the remnant of the cosmic recombination epoch. It contains abundant information of the early universe. The positions of the acoustic peaks contains the information of dark energy (Peebles and Yu 1970; Bond and Efstathiou 1984). The shift parameter is defined as (Bond et al. 1997; Wang and Mukherjee 2006)

$$
\mathcal{R}=\frac{\sqrt{\Omega_{M}}}{\sqrt{\left|\Omega_{k}\right|}} \operatorname{sinn}\left(\sqrt{\left|\Omega_{k}\right|} \int_{0}^{z_{\mathrm{ls}}} \frac{d z}{E(z)}\right)=1.70 \pm 0.03
$$


where $E(z) \equiv H(z) / H_{0}$ and the function $\operatorname{sinn}(x)$ is defined as $\operatorname{sinn}(x)=\sin (x)$ for a closed universe, $\operatorname{sinn}(x)=\sinh (x)$ for an open universe and $\operatorname{sinn}(x)=x$ for a flat universe. The last scattering redshift $z_{\text {ls }}$ can be fitted as

$$
z_{\mathrm{ls}}=1048\left[1+0.00124\left(\Omega_{b} h^{2}\right)^{-0.738}\right]\left[1+g_{1}\left(\Omega_{M} h^{2}\right)^{g_{2}}\right],
$$

where the quantities $g_{1}$ and $g_{2}$ are defined as $g_{1}=$ $0.078\left(\Omega_{b} h^{2}\right)^{-0.238}\left[1+39.5\left(\Omega_{b} h^{2}\right)^{0.763}\right]^{-1}$ and $g_{2}=0.56[1+$ $\left.21.1\left(\Omega_{b} h^{2}\right)^{1.81}\right]^{-1}$ respectively (Hu and Sugiyama 1996). The $\chi^{2}$ value is

$$
\chi_{\mathrm{CMB}}^{2}=\frac{(\mathcal{R}-1.70)^{2}}{0.03^{2}} .
$$

BAO refers to an overdensity of baryonic matter due to acoustic waves which propagated in the early universe Silk 1968; Peebles and Yu 1970). BAO provides a "standard ruler" for length scale in cosmology to explore the expansion history of the universe. The acoustic signatures in the large-scale clustering of galaxies can be used to constrain cosmological parameters by detection of a peak in the correlation function (Eisenstein et al. 2005). The $A$ parameter is defined as

$$
A=\frac{\sqrt{\Omega_{M}}}{z_{1}}\left[\frac{z_{1}}{E\left(z_{1}\right)} \frac{1}{\left|\Omega_{k}\right|} \operatorname{sinn}^{2}\left(\sqrt{\left|\Omega_{k}\right|} \int_{0}^{z_{1}} \frac{d z}{E(z)}\right)\right]^{1 / 3},
$$

measured from the SDSS data to be $A=0.469(0.95 / 0.98)^{-0.35} \pm$ 0.017 , where $z_{1}=0.35$. The $\chi^{2}$ value is

$$
\chi_{\mathrm{BAO}}^{2}=\frac{(A-0.469)^{2}}{0.017^{2}}
$$

In Figure 5, the constraint on the $\Lambda \mathrm{CDM}$ model is shown. Different color contours represent constraints from different data. The best fitted parameters are consistent with a flat geometry. Figure 6 shows the cosmological constrain on the $w=w_{0}$ model from SNe Ia (blue) and GRBs (red). The combined constraint is shown as solid contours. The constraint is much tighter by adding the GRB data. Li et al. (2008b) also performed a Markov Chain Monte Carlo (MCMC) global fitting analysis to overcome the circularity problem. The Ghirlanda correlation and 27 GRBs are used. They treated the slopes of Ghirlanda correlation and cosmological parameters as free parameters and determine them simultaneously through MCMC analysis on GRB data together with other observational data, such as SNe Ia, CMB and large-scale structure (LSS). Amati et al. (2008) measured the cosmological parameters using Amati correlation using global fitting method. The extrinsic scatter was assumed on the parameter of $E_{\text {peak }}$, but the cosmological-dependent value is $E_{\text {iso }}$ (Ghirlanda 2009).

The second method is to calibrate the correlations of GRBs using SNe Ia data at low redshifts. The principle of this method is that objects at the same redshift should have the same luminosity distance in any cosmology model. Therefore, the luminosity distance at any redshift in the redshift range of GRBs can be obtained by interpolating (or by other approaches) directly from the SNe Ia Hubble diagram. Then if further assuming these calibrated GRB correlations to be valid for all long GRB

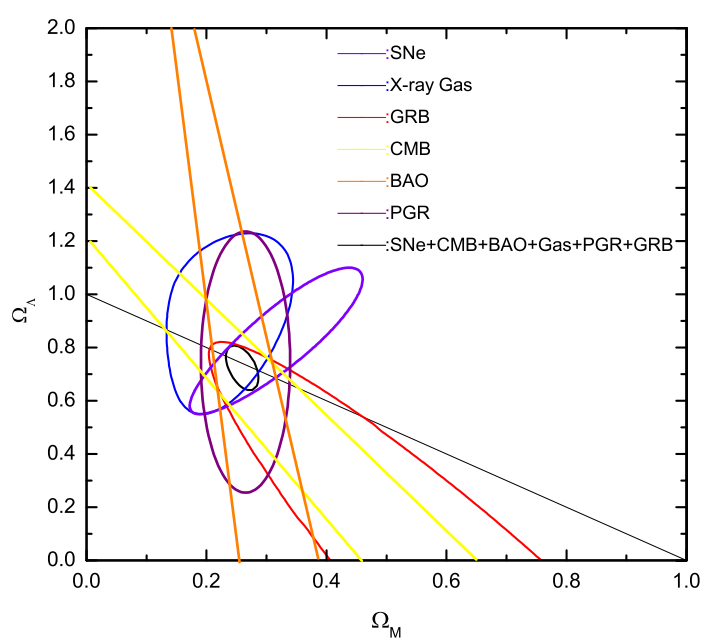

Figure 5: Joint confidence intervals of $1 \sigma$ for $\left(\Omega_{M}, \Omega_{\Lambda}\right)$ from the observational datasets. The thick black line contour, the blue contour, the red contour, the yellow contour, the violet contour, the orange contour, and the purple contour show constraints from from all the datasets, 26 galaxy clusters, 69 GRBs, CMB shift parameter, 182 SNe Ia, BAO, and 2dF Galaxy Redshift Survey, respectively. The thin solid line represents a flat universe. (Adapted from Figure 2 in Wang et al. (2007).)

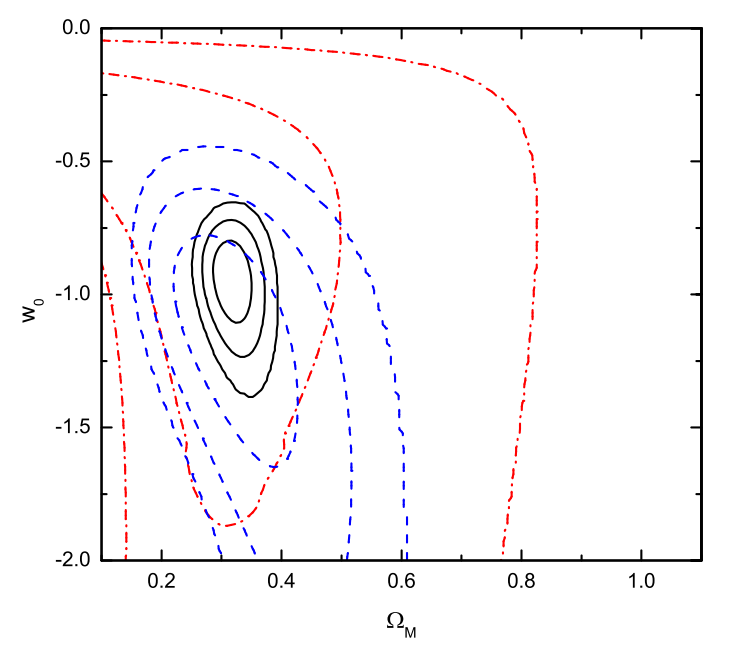

Figure 6: Joint confidence intervals of $1 \sigma$ to $3 \sigma$ for $\left(\Omega_{M}, w_{0}\right)$ from the observational datasets. The solid contours, the dashed blue contours, and the dotdashed red contours show constraints from all the datasets, 26 galaxy clusters, and 69 GRBs, respectively. (Adapted from Figure 4 in Wang et al. (2007).) 
data, the standard Hubble diagram method to constrain the cosmological parameters from the GRB data at high redshifts obtained by utilizing the correlations. Liang et al. (2008) first calibrated the GRB correlations using an interpolation method. The error of distance modulus of linear interpolation can be calculated as (Liang et al. 2008; Wei 2010)

$\sigma_{\mu}=\left(\left[\left(z_{i+1}-z\right) /\left(z_{i+1}-z_{i}\right)\right]^{2} \epsilon_{\mu, i}^{2}+\left[\left(z-z_{i}\right) /\left(z_{i+1}-z_{i}\right)\right]^{2} \epsilon_{\mu, i+1}^{2}\right)^{1 / 2}$,

where $\epsilon_{\mu, i}$ and $\epsilon_{\mu, i+1}$ are errors of the SNe Ia, $\mu_{i}$ and $\mu_{i+1}$ are the distance moduli of the SNe Ia at $z_{i}$ and $z_{i+1}$ respectively. Similar to the interpolation method, Cardone et al. (2009) constructed an updated GRBs Hubble diagram on six correlations calibrated by local regression from SNe Ia. Kodama et al. (2008) presented that the $L_{\text {iso }}-E_{\text {peak }}$ correlation can be calibrated with the empirical formula fitted from the luminosity distance of SNe Ia. This method has been used to constrain cosmological parameters by combining these GRB data with $\mathrm{SNe}$ Ia in a following work by Tsutsui et al. (2008).

However, it must be noted that this calibration procedure depends seriously on the choice of the formula and various possible formulas can be fitted from the SNe Ia data that could give different calibration results of GRBs. As the cosmological constraints from GRBs are sensitive to GRBs calibration results (Wang 2008), the reliability of this method should be tested carefully. Moreover, as pointed out by Wang (2008), the GRB luminosity correlations which are calibrated by this way are no longer completely independent of all the SNe Ia data points. Therefore these GRB data can not be used to directly combine with the whole SNe Ia dataset to constrain cosmological parameters and dark energy. In order to search a unique expression of the fitting formula, Wang et al. (2009a) used the cosmographic parameters (Capozziello and Izzo 2008; Vitagliano et al. 2010; Xia et al. 2012; Gao et al. 2011). The luminosity distance can be expanded as (Visser 2004)

$$
\begin{gathered}
d_{L}=\frac{c}{H_{0}}\left\{z+\frac{1}{2}\left(1-q_{0}\right) z^{2}-\frac{1}{6}\left(1-q_{0}-3 q_{0}^{2}+j_{0}\right) z^{3}\right. \\
\left.+\frac{1}{24}\left[2-2 q_{0}-15 q_{0}^{2}-15 q_{0}^{3}+5 j_{0}+10 q_{0} j_{0}+s_{0}\right] z^{4}+O\left(z^{5}\right)\right\}
\end{gathered}
$$

where $q$ is the deceleration parameter, $j$ is the so-called "jerk", and $s$ is the so-called "snap" parameter. These quantities are defined as

$$
\begin{gathered}
q=-\frac{1}{H^{2}} \frac{\ddot{a}}{a} ; \\
j=\frac{1}{H^{3}} \frac{\dot{a}}{a} ; \\
s=\frac{1}{H^{4}} \frac{\ddot{a}}{a} .
\end{gathered}
$$

Equation (20) is only dependent on the cosmological principles and FRW metric, so the expansion is model-independent. But the Taylor-expansion of $d_{L}$ is not valid at $z>1$. So expansion $d_{L}$ as a function of $y=z / 1+z$ is much useful (Cattoën and Visser 2007; Wang and Dai 2011b). The calibrated Hubble diagram of GRBs is shown in Figure7 (Wang and Dai 2011b).

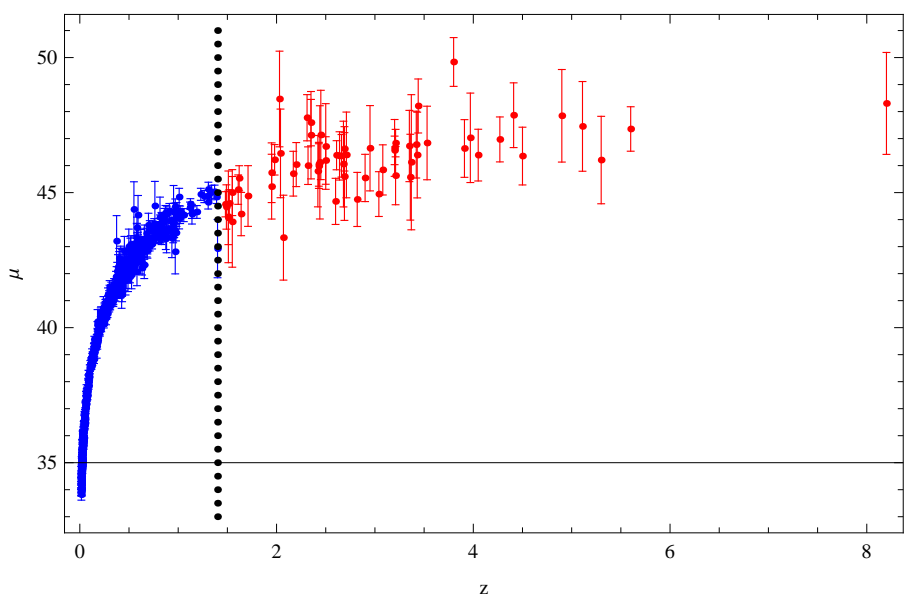

Figure 7: The Hubble diagram of 557 SNe Ia (blue) and 66 high-redshift GRBs (red). (Adapted from Figure 2 in Wang and Dai (2011b).)

The third method is to calibrate the standard candles using GRBs in a narrow redshift range $(\delta z)$ near a fiducial redshift (Lamb et al. 2005; Liang and Zhang 2006; Ghirlanda et al. 2006b). Liang and Zhang (2006) proposed a procedure to calibrate the Liang-Zhang correlation with a sample of GRBs in a narrow redshift range. No low-redshift GRB sample is needed in this method. The calibration procedure can be described as follows. First, calibrate the power-law index of Liang-Zhang correlation using a sample of GRBs that satisfy this correlation and are distributed in a narrow redshift range. The power-law index can be derived using a multiple regression method. Second, marginalize the coefficient value over a reasonable range.

However, the gravitational lensing by random fluctuations in the intervening matter distribution induces a dispersion in GRB brightness (Oguri and Takahashi 2006; Schaefer 2007), degrading their value as standard candles as well as $\mathrm{SNe}$ Ia ( $\mathrm{Holz}$ 1998). GRBs can be magnified (or reduced) by the gravitational lensing produced by the structure of the Universe. The gravitational lensing has sometimes a great impact on high-redshift GRBs. First, the probability distribution functions (PDFs) of gravitational lensing magnification have much higher dispersions and are markedly different from the Gaussian distribution (Valageas 2000; Oguri and Takahashi 2006; Wang and Dai 2011b). Figure 8 shows the magnification probability distribution functions of gravitational lensing at different redshifts (Wang and Dai 2011b). Second, there is effectively a threshold for the detection in the burst apparent brightness. With gravitational lensing, bursts just below this threshold might be magnified in brightness and detected, whereas bursts just beyond this threshold might be reduced in brightness and excluded. Wang and Dai (2011b) considered the weak lensing effect on cosmological parameters derived from GRBs, and found that the most probable value of the observed matter density $\Omega_{M}$ is slightly lower than its actual value, see Figure 9. The weak gravitational lensing also affects the dark energy equation of state by shifting it to a more negative value. 


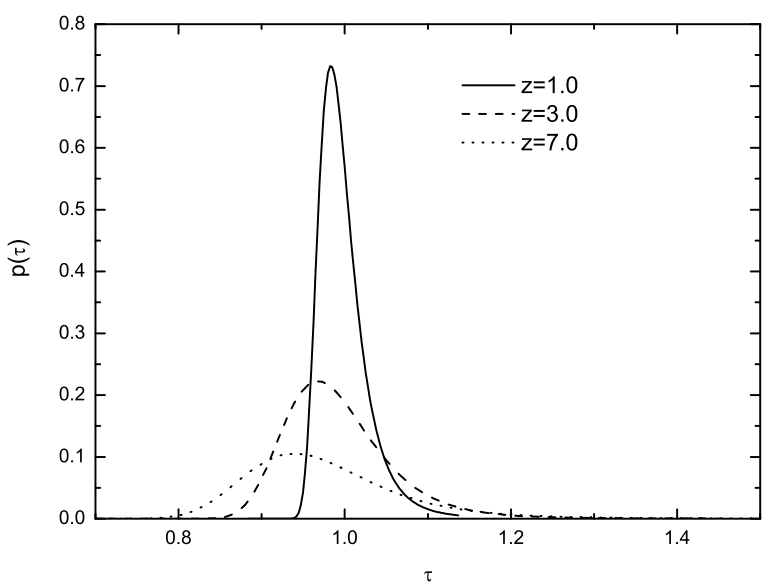

Figure 8: Magnification probability distribution functions of gravitational lensing at redshifts $z=1, z=3$ and $z=7$. (Adapted from Figure 5 in Wang and Dai (2011b).)

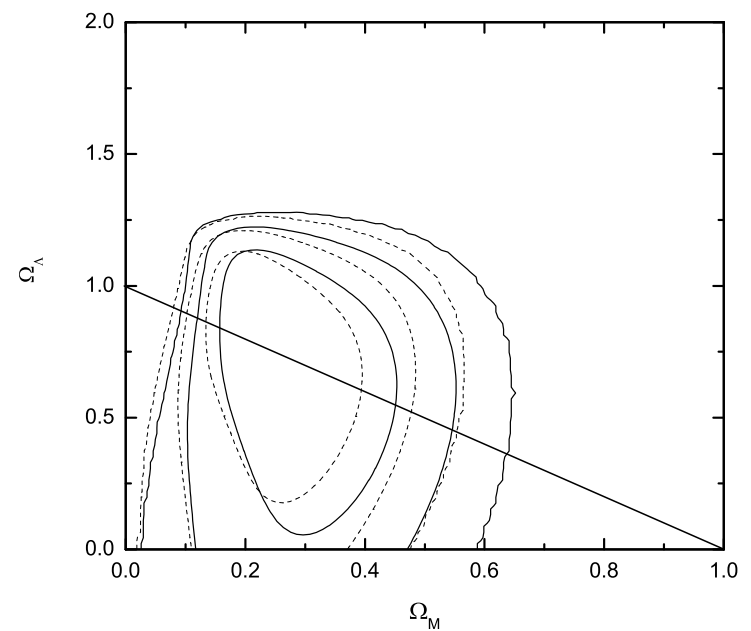

Figure 9: Confidence contours of likelihood from $1 \sigma$ to $3 \sigma$ in the $\Lambda$ CDM model. The black line contours from $116 \mathrm{GRBs}$ and the dotted contours from 116 GRBs including magnification bias. (Adapted from Figure 7 in Wang and Dai (2011b).)

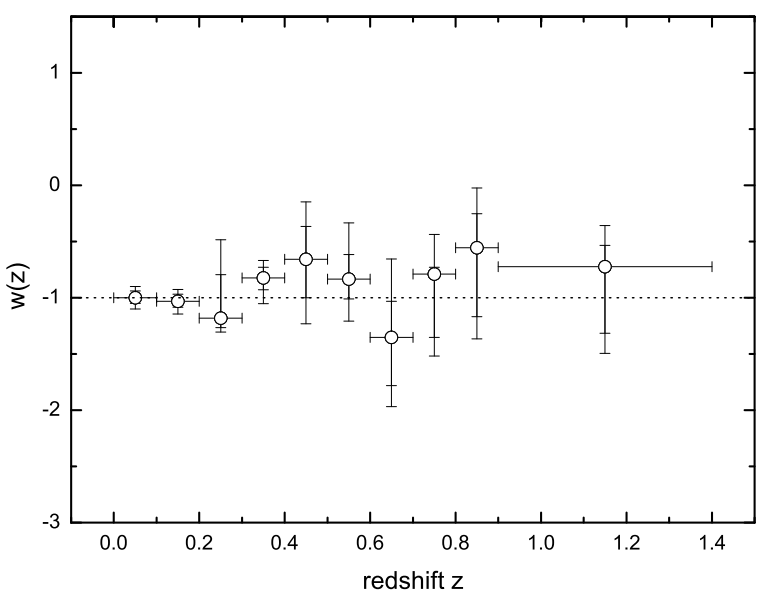

Figure 10: Estimation of the uncorrelated dark energy EOS parameters at different redshift bins $\left(w_{1}, w_{2}, \ldots, w_{10}\right)$ from $\mathrm{SNe} \mathrm{Ia}+\mathrm{BAO}+\mathrm{WMAP} 9+\mathrm{H}(\mathrm{z})+\mathrm{GRB}$ data. The open points show the best fit value. The error bars are $1 \sigma$ and $2 \sigma$ confidence levels. The dotted line shows the cosmological constant. (Adapted from Figure 3 in Wang and Dai (2014a).)

\subsection{The equation of state of dark energy}

The dark energy equation of state $w$ is the most important parameter that describes the properties of dark energy. Whether and how it evolves with time is crucial for revealing the physics of dark energy. GRBs can provide the high-redshift evolution property of dark energy. The procedure is to bin $w$ in $z$, and fit the $w$ in each bin to observational data by assuming that $w$ is constant in each bin. The function $f(z)$ in equation (7) should be described as

$$
f\left(z_{n-1}<z \leq z_{n}\right)=(1+z)^{3\left(1+w_{n}\right)} \prod_{i=0}^{n-1}\left(1+z_{i}\right)^{3\left(w_{i}-w_{i+1}\right)},
$$

where $w_{i}$ is the EOS parameter in the $i^{\text {th }}$ redshift bin defined by an upper boundary at $z_{i}$, and the zeroth bin is defined as $z_{0}=0$. Oi et al. (2008a) used GRBs and other cosmological observations to construct evolution of the equation of state, and found that the equation of state $w$ is consistent with the cosmological constant (also see Qi et al. 2008b). The confidence interval of the uncorrelated equation of state parameter can be significantly reduced by adding GRBs. After calibrating the GRB correlations using cosmographic parameters, Wang and Dai (2011b) found that the high-redshift $(1.4<z<8.2)$ equation of state is consistent with the cosmological constant. But some studies found that the equation of state $w$ may deviate from -1 (i.e., Qi et al. 2009; Zhao et al. 2012). In light of the Planck CMB data, Wang and Dai (2014a) found that the EOS is consistent with the cosmological constant at the $2 \sigma$ confidence level, not preferring to a dynamical dark energy, which is shown in Figure 10. 


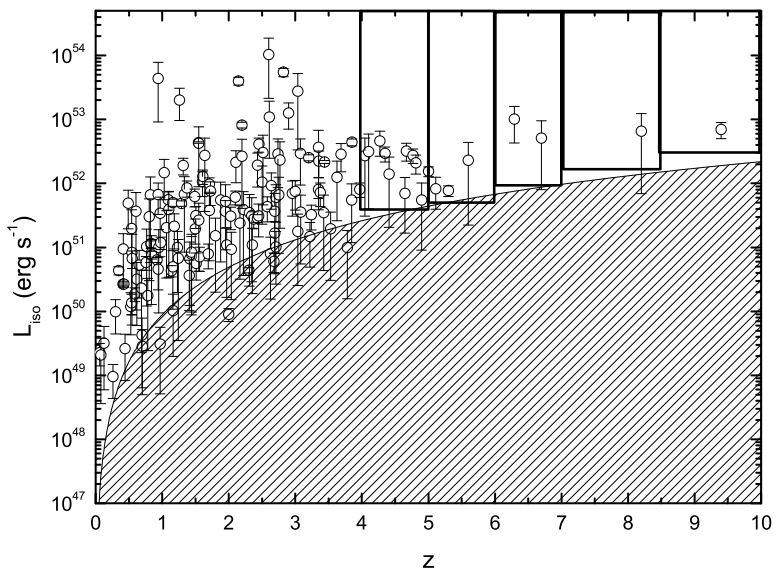

Figure 11: Distribution of the isotropic-equivalent luminosity for 157 longduration Swift GRBs. The shaded area approximates the detection threshold of Swift BAT. (Adapted from Figure 1 in Wang (2013).)

\section{Probing the star formation rate}

\subsection{Star formation rate derived from GRBs}

The association of long GRBs with core-collapse supernovae has been confirmed from observations in recent years (Stanek et al. 2003; Hjorth et al. 2003), which provides a complementary technique for measuring the high-redshift SFR (Totani 1997; Wijers et al. 1998; Lamb and Reichart 2000; Porciani and Madau 2001; Bromm and Loeb 2002). The selection effects should be considered (for a review, see Coward 2007). But one crucial problem appears, i.e., how to calibrate the GRB event rate to the SFR. The luminosity function may play an important role (Nataraian et al. 2005; Daigne et al. 2006; Salvaterra and Chincarini 2007; Salvaterra et al. 2009; Campisi et al. 2010; Wanderman and Piran 2010; Cao et al. 2011). Before the launch of Swift (Gehrels et al. 2004), the luminosity function is determined by fitting the observed $\log N-$ $\log P$ distribution (Schmidt 1999; Porciani and Madau 2001; Guetta et al. 2005; Natarajan et al. 2005). Thanks to the Swift, more redshifts of GRBs are measured. This makes it possible to give more information on the luminosity function (Wanderman and Piran 2010; Cao et al. 2011; Tan et al. 2013). Because the form of luminosity function should be assumed and the model parameters of luminosity function is degenerate, it is not easy to determine the luminosity function. A straightforward way to estimate the luminosity function is proposed by Lynden-Bell (1977) and then further developed by Efron and Petrosian (1992). This method has been used for GRBs (Llyd-Ronning et al. 2002; Yonetoku et al. 2004; Wu et al. 2012). There are two luminosity function models in the literature, a broken power law and a single power law with an exponential cut-off at low luminosities.

In order to avoid the poorly known luminosity function when studying high-redshift SFR, a method that only high-luminosity GRBs are used is proposed (Yüksel et al. 2008; Kistler et al.
2009; Wang and Dai 2009; Yu et al. 2012; Wang 2013). The expected redshift distribution of GRBs is

$$
\frac{d N}{d z}=F(z) \frac{\varepsilon(z) \dot{\rho}_{*}(z)}{\left\langle f_{\text {beam }}\right\rangle} \frac{d V_{\text {com }} / d z}{1+z},
$$

where $F(z)$ represents the ability to obtain the redshift, $\varepsilon(z)$ accounts for the fraction of stars producing GRBs, and $\dot{\rho}_{*}(z)$ is the SFR density. The $F(z)$ can be treated as constant when we consider the bright bursts with luminosities sufficient to be detected within an entire redshift range. GRBs that are unobservable due to beaming are accounted for through $\left\langle f_{\text {beam }}\right\rangle$. The $\varepsilon(z)$ can be parameterized as $\varepsilon(z)=\varepsilon_{0}(1+z)^{\delta}$, where $\varepsilon_{0}$ is an unknown constant that includes the absolute conversion from the SFR to the GRB rate in a given GRB luminosity range. Kistler et al. (2008) found the index $\delta=1.5$ from 63 Swift GRBs. A little smaller value $\delta \sim 0.5-1.2$ has been inferred from update $S$ wift GRBs (Kistler et al. 2009; Wang 2013). In a flat universe, the comoving volume is calculated by

$$
\frac{d V_{\mathrm{com}}}{d z}=4 \pi D_{\mathrm{com}}^{2} \frac{d D_{\mathrm{com}}}{d z},
$$

where the comoving distance is

$$
D_{\text {com }}(z) \equiv \frac{c}{H_{0}} \int_{0}^{z} \frac{d z^{\prime}}{\sqrt{\Omega_{m}\left(1+z^{\prime}\right)^{3}+\Omega_{\Lambda}}} .
$$

In the calculations, the $\Lambda$ CDM model with $\Omega_{m}=0.27$, $\Omega_{\Lambda}=0.73$ and $H_{0}=71 \mathrm{~km} \mathrm{~s}^{-1} \mathrm{Mpc}^{-1}$ from the Wilkinson $\mathrm{Mi}$ crowave Anisotropy Probe (WMAP) seven-year data is used (Komatsu et al. 2011).

Figure 11 shows the isotropic luminosity distribution of 157 Swift GRBs. The isotropic luminosity can be obtained by

$$
L_{\text {iso }}=E_{\text {iso }}(1+z) / T_{90}
$$

where $T_{90}$ is the duration time. The shaded area approximates the detection threshold of Swift BAT, which has a flux limit $\sim F_{\lim }=1.2 \times 10^{-8} \mathrm{erg} \mathrm{cm}^{-2} \mathrm{~s}^{-1}$. So the selection effect is important. In order to exclude faint low -redshift GRBs that could not be visible at high redshifts, we only select luminous bursts. The luminosity cut $L_{\text {iso }}>10^{51} \mathrm{erg} \mathrm{s}^{-1}$ is chosen in the redshift bin $0-4$ (Yüksel et al. 2008), which removes many low-redshift, low-luminosity bursts that could not be detected at higher redshift. The cumulative distribution of GRB redshift can be expressed as

$$
\frac{N(<z)}{N\left(<z_{\max }\right)}=\frac{N(0, z)}{N\left(0, z_{\max }\right)} .
$$

The value of $z_{\max }$ is taken as 4.0. Because the SFR has been well measured at $z<4.0$ (Hopkins and Beacom 2006). The theory predicted and observed cumulative GRB distributions is shown in Figure 12. The Kolmogorov-Smirnov statistic gives the minimization for $\delta=0.5$ Wang 2013). At the $2 \sigma$ confidence level, the value of $\delta$ is in the range $-0.15<\delta<1.6$.

There are four redshift bins, $z=4-5,5-6,6-7,7-8.5$ and $8.5-10$. The GRBs in $z=1-4$ play as a "control group" to 


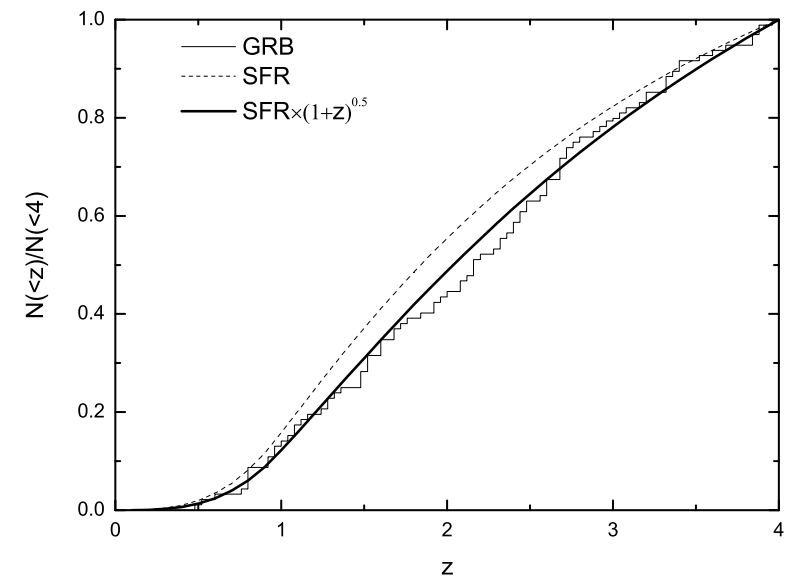

Figure 12: Cumulative distribution of 92 Swift GRBs with $L_{\text {iso }}>10^{51} \mathrm{erg} \mathrm{s}^{-1}$ in $z=0-4$ (stepwise solid line). The dashed line shows the GRB rate inferred from the star formation history of Hopkins \& Beacom (2006). The solid line shows the GRB rate inferred from the star formation history including $(1+z)^{0.5}$ evolution. (Adapted from Figure 2 in Wang (2013).)

constrain the GRB-to-SFR conversion. The theoretically predicated number of GRBs in this bin can be calculated as

$$
\begin{aligned}
N_{1-4}^{\mathrm{th}} & =\Delta t \frac{\Delta \Omega}{4 \pi} \int_{1}^{4} d z F(z) \varepsilon(z) \frac{\dot{\rho}_{*}(z)}{\left\langle f_{\text {beam }}\right\rangle} \frac{d V_{\mathrm{com}} / d z}{1+z} \\
& =A \int_{1}^{4} d z(1+z)^{\delta} \dot{\rho}_{*}(z) \frac{d V_{\mathrm{com}} / d z}{1+z}
\end{aligned}
$$

where $A=\Delta t \Delta \Omega F_{0} / 4 \pi\left\langle f_{\text {beam }}\right\rangle$ depends on the total observed time of Swift, $\Delta t$, and the angular sky coverage, $\Delta \Omega$. The theoretical number of GRBs in redshift bin $z_{1}-z_{2}$ is

$$
N_{z_{1}-z_{2}}^{\mathrm{th}}=\left\langle\dot{\rho}_{*}\right\rangle_{z_{1}-z_{2}} A \int_{z_{1}}^{z_{2}} d z(1+z)^{\delta} \frac{d V_{\mathrm{com}} / d z}{1+z},
$$

where $\left\langle\dot{\rho}_{*}\right\rangle_{z_{1}-z_{2}}$ is the SFR in the redshift range $z_{1}-z_{2}$. Representing the predicated numbers, $N_{z_{1}-z_{2}}^{\text {th }}$ with the observed GRB counts, $N_{z_{1}-z_{2}}^{\text {obs }}$, we obtain the SFR in the redshift range $z_{1}-z_{2}$,

$$
\left\langle\dot{\rho}_{*}\right\rangle_{z_{1}-z_{2}}=\frac{N_{z_{1}-z_{2}}^{\mathrm{obs}}}{N_{1-4}^{\mathrm{obs}}} \frac{\int_{1}^{4} d z \frac{d V_{\mathrm{com}} / d z}{1+z}(1+z)^{\delta} \dot{\rho}_{*}(z)}{\int_{z_{1}}^{z_{2}} d z \frac{d V_{\mathrm{com}} / d z}{1+z}(1+z)^{\delta}} .
$$

The derived SFR from GRBs are shown as filled circles in Figure 13. Error bars correspond to $68 \%$ Poisson confidence intervals for the binned events (Gehrels 1986). The high-redshift SFRs obviously decrease with increasing redshifts, although an oscillation may exist. The SFRs from GRBs are dramatically larger than those from other observations. The main reason is that other observations probe only the brightest galaxies, especially at high redshifts. But GRBs can reveal the faint galaxies at high redshifts due to their high luminosity. The SFR at $z>4.48$ is proportional to $(1+z)^{-3}$, which is shown as solid line in Figure 13

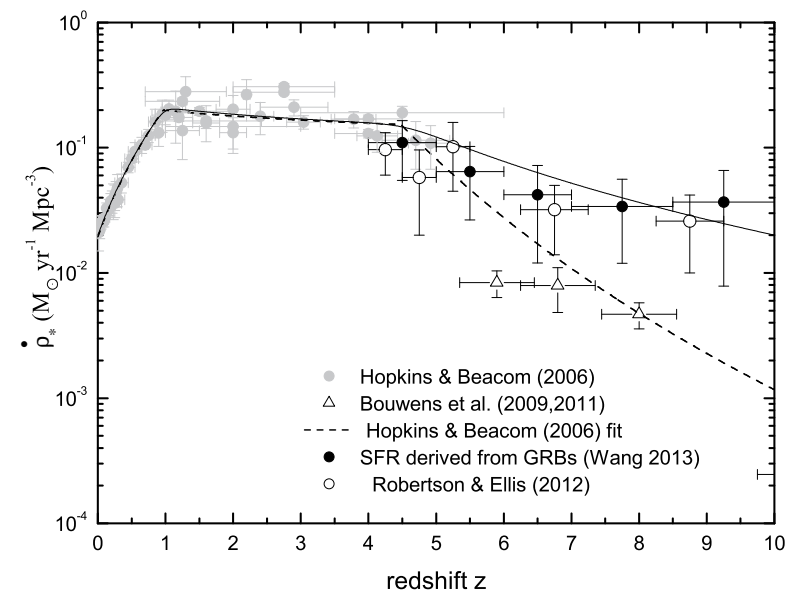

Figure 13: The cosmic star formation history. The grey points are taken from Hopkins \& Beacom (2006), the dashed line shows their fitting result. The triangular points are from Bouwens et al. $(2009,2011)$. The open circles are taken from Robertson and Ellis (2012). The filled circles are the SFR derived from GRBs in Wang (2013). (Adapted from Figure 3 in Wang (2013).)

\subsection{Possible origins of high-redshift GRB rate excess}

Recent studies show that the rate of GRBs does not strictly follow the SFH but is actually enhanced by some mechanism at high redshift (Le and Dermer 2007; Salvaterra and Chincarini 2007; Kistler et al. 2008; Yüksel et al.2008; Wang et al. 2009b; Robertson and Ellis 2012; Wang 2013). The SFR inferred from the high-redshift $(z>6)$ GRBs seems to be too high in comparison with the SFR obtained from some high-redshift galaxy surveys (Bouwens et al. 2009, 2011).

\subsubsection{Metallicity evolution}

A natural origin of the high-redshift GRB rate excess is the metallcity evolution. Theory and observation both support that long GRBs prefer to occurring in low-metallicity environment. Some theoretical studies of long GRBs progenitors using stellar evolution models suggest that low metallicity may be a necessary condition for a long GRB to occur. For popular collapse models of long GRBs, stars with masses $>30 M_{\odot}$ can be able to create a black hole $(\mathrm{BH})$ remnant (Woosley 1993; Hirschi et al. 2005). The preservation of high angular momentum and high-stellar mass at the time of collapse (Woosley 1993; MacFadyen and Woosley 2006) is crucial for producing a relativistic jet and high luminosity. Low-metallicity $(0.1-$ $0.3 Z_{\odot}$ ) progenitors can theoretically retain more of their mass due to smaller line-driven stellar winds Kudritzki and Puls 2000; Vink and de Koter 2005), and hence preserve their angular momentum (Yoon and Langer 2005; Yoon et al. 2006; Woosley and Heger 2006), because the wind-driven mass loss of massive stars is proportional to the metallicity. Observations of long GRB host galaxies also show that they are typically in low metallicity environment, for several local long GRB host galaxies (Sollerman et al. 2006; Stanek et al. 2006), 
as well as in distant long GRB hosts (i.e., Fruchter et al. 2006; Prochaska et al. 2007).

Li (2008) studied the possibility of interpreting the observed discrepancy between the GRB rate history and the star formation rate history using cosmic metallicity evolution (Kistler et al. 2008). Under the assumption that the formation of long GRBs follows the cosmic star formation history and form preferentially in low-metallicity galaxies, the rate of GRB is given by

$$
R_{\mathrm{GRB}}(z)=k_{\mathrm{GRB}} \Sigma\left(Z_{\mathrm{th}}, z\right) \rho_{*}(z),
$$

where $k_{\mathrm{GRB}}$ is the GRB formation efficiency, $\Sigma\left(Z_{\mathrm{th}}, z\right)$ is the fraction of galaxies at redshift $z$ with metallicity below $Z_{\text {th }}$ (Langer and Norman 2006) and $\rho_{*}(z)$ is the observed SFR. The function $\Sigma\left(Z_{\mathrm{th}}, z\right)$ is (Langer and Norman 2006)

$$
\Sigma\left(Z_{\mathrm{th}}, z\right)=\frac{\hat{\Gamma}\left[\alpha_{1}+2,\left(Z_{\mathrm{th}} / Z_{\odot}\right)^{2} 10^{0.15 \beta z}\right]}{\Gamma\left(\alpha_{1}+2\right)}
$$

where $\hat{\Gamma}$ and $\Gamma$ are the incomplete and complete gamma functions, $\alpha_{1}=-1.16$ and $\beta=2$ (Savaglio et al. 2005). Li (2008) found that the distribution of luminosity and cumulative distribution of redshift could be well fitted if $Z_{\text {th }}=$ $0.3 Z_{\odot}$ is adopted. Wang and Dai (2009) studied the highredshift SFR by considering the GRBs tracing the star formation history and the cosmic metallicity evolution. They found the SFR derived from GRBs is marginal consistent with that from traditional way (i.e., Hopkins and Beacom 2006). Using Monte Carlo simulations, Oin et al. (2010) compared the simulation results to the Swift observations with $\log N-\log P$ and luminosity-redshift distributions. They found that the observed distributions are well consistent with that from simulations if the GRB rate is proportional to the SFR incorporating with the cosmic metallicity history with $Z_{\text {th }}=0.6 Z_{\odot}$. Figure 14 shows the comparison between simulations and observation. Wei et al. (2014) examined the influence on the GRB distribution due to the background cosmology, i.e., $R_{h}=c t$ Universe. However, a few GRB hosts with high metallicity are observed (i.e. GRB 020819), so that the role of metallicity in driving the GRB phenomena remains unclear and it is still debated (Price et al. 2007; Wolf and Podsiadlowski 2007; Kocevski et al. 2009; Graham et al. 2009; Svensson et al. 2010). For excellent reviews, see Fynbo et al. (2012) and Levesque (2014). But there are some uncertainties when measure the metallicities of GRBs' explosion region at highredshifts, such as chemical inhomogeneity (Levesque et al. 2010; Niino 2011). Wang and Dai (2014b) studied the metallicity role from two aspects, the GRB host galaxies and redshift distribution. They found that the the observed GRB host galaxy masses and the cumulative redshift distribution can fit the predicted distributions well if GRBs occur in lowmetallicity $12+\log (\mathrm{O} / \mathrm{H})_{\mathrm{KK} 04}<8.7$, which is shown in Figure 15. Trenti et al. (2015) found that there is clear evidence for a relation between SFR and GRB (Jimenez and Piran 2013). But a sharp cut-off of metallicity is ruled out.

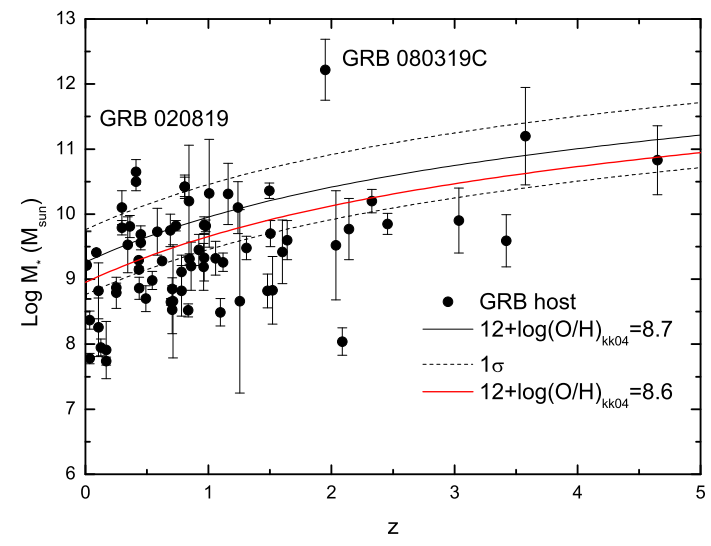

Figure 15: GRB host galaxy mass distribution. The solid lines represent the upper limits of the stellar mass of a GRB host galaxy given a metallicity cutoff of $12+\log (\mathrm{O} / \mathrm{H})_{\mathrm{KK} 04}=8.7$ (black), and $12+\log (\mathrm{O} / \mathrm{H})_{\mathrm{KK} 04}=8.6$ (red). The dashed lines represent the $1 \sigma$ scatter. (Adapted from Figure 4 in Wang and Dai (2014b).)

\subsubsection{Evolving star initial mass function}

Wang and Dai (2011a) proposed that the GRB rate excess may be due to the evolution of star initial mass function (IMF), also see (Xu and Wei 2008). Because an "top-heavy" IMF will lead to more massive stars at high-redshift which can result in much more GRBs. Considering long GRBs trace SFR, the rate of GRBs in an evolving IMF is

$$
R_{\mathrm{GRB}} \propto \frac{N_{m>30 M_{\odot}}}{V}=K\left(\frac{c}{H_{0}}\right)^{-3} \frac{\int_{30 M_{\odot}}^{m_{l}} \xi(m) d \log m}{\int_{m_{s}}^{m_{l}} m \xi(m) d \log m} \rho_{*}(z),
$$

where $K$ is a constant to be constrained and $R_{\mathrm{GRB}}$ is the rate of GRBs, representing the number of GRBs per unit time per unit volume at redshift $z$. The evolving IMF proposed by Dave (2008) is

$$
\frac{d N}{d \log m}=\xi(m) \propto \begin{cases}m^{-0.3} & \text { for } m<\hat{m}_{\mathrm{IMF}} \\ m^{-1.3} & \text { for } m>\hat{m}_{\mathrm{IMF}},\end{cases}
$$

where $\hat{m}_{\mathrm{IMF}}=0.5(1+z)^{2} M_{\odot}$, which has been constrained by requiring non-evolving star formation activity parameter. Figure 16 shows that the observed cumulative distribution of GRBs can be well produced by this model.

\subsubsection{Evolving luminosity function break}

Virgili et al. (2011) found that if the break of luminosity function evolves with redshift, the distributions of luminosity, redshift and peak photon flux from the BATSE and Swift data can be reproduced from simulations. The break luminosity function evolution can be in a moderate way $\propto L_{b} \times(1+z)^{\sim 0.8-1.2}$. Campisi et al. (2010) studied the luminosity function, the rate of long GRBs at high redshift, using high-resolution N-body simulations. A strongly evolving luminosity function with no metallicity cut may well explain the $\log N-\log P$ distribution of BATSE and Swift data. 

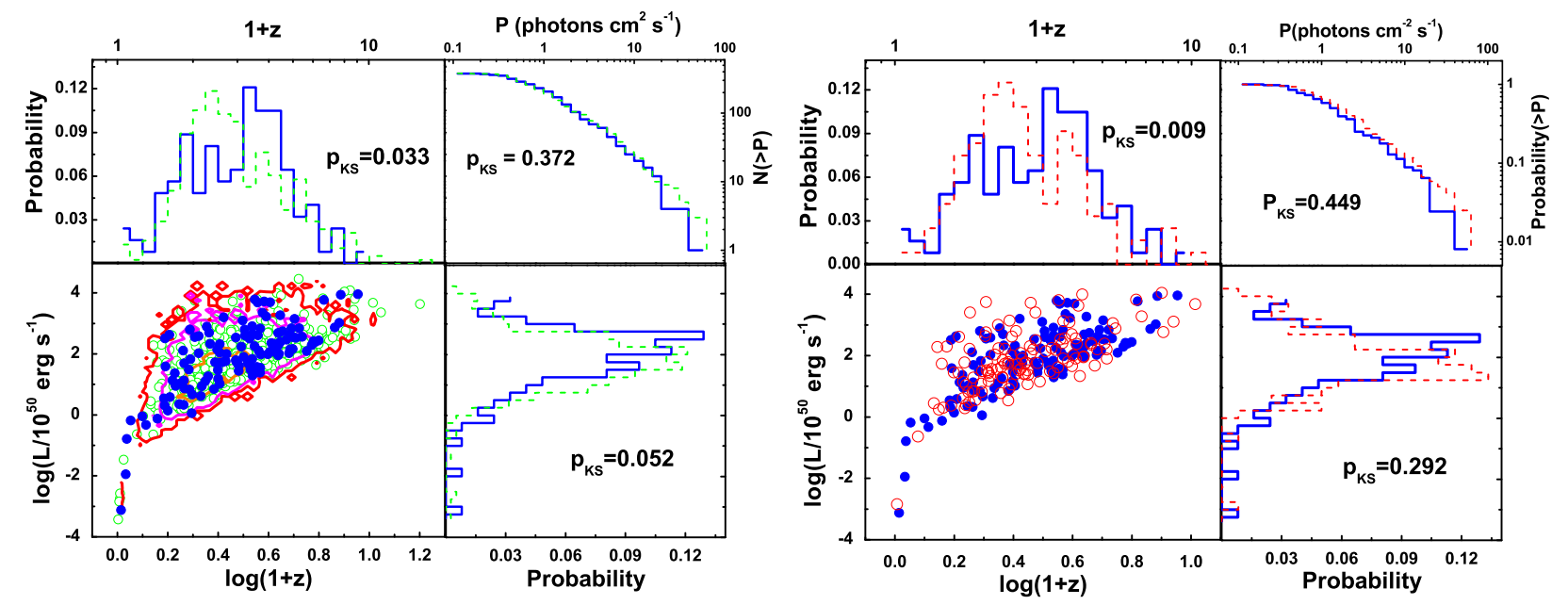

Figure 14: Comparisons of $\log L-\log z$ distributions and $\log L, \log z$, and $\log P$ distributions between the observed Swift/BAT GRB sample (solid) and simulations (open dots and dashed lines) for: $R_{\mathrm{GRB}}(z) \propto \rho_{*}(z) \times \Sigma\left(Z_{\mathrm{th}}, z\right)$. left four panels are for the trigged GRB sample. Right four panels are for the sample with redshift measurement. One dimensional K-S test probabilities for the comparisons are presented in each panel. (Adapted from Figure 5 in Qin et al. (2010).)

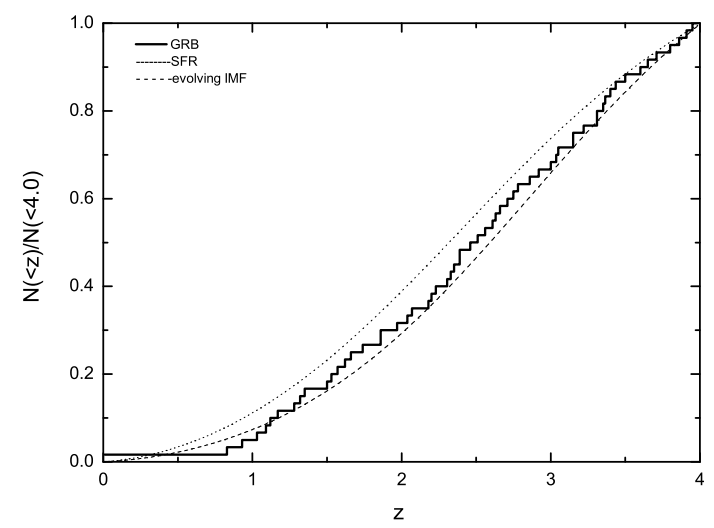

Figure 16: The cumulative distribution of 72 Swift long GRBs with $L_{\text {iso }}>$ $0.8 \times 10^{51} \mathrm{erg} \mathrm{s}^{-1}$ (stepwise solid line). The dotted line shows the GRB rate inferred from the star formation history of Hopkins \& Beacom (2006). The dashed line shows the GRB rate inferred from star formation history including an evolving IMF. (Adapted from Figure 2in Wang and Dai (2011a).)

\subsubsection{Superconducting cosmic string}

Cosmic strings are thought to be linear topological defects that could be formed at a phase transition in very early Universe. By considering that high-redshift GRBs 080913 and 090423 are electromagnetic bursts of superconducting cosmic strings, Cheng et al. (2010) showed the high-redshift GRB excess can be reconciled. But Wang et al. (2011) claimed that GRBs from cosmic string have a very small angle, about $10^{-3}$, which could be in contradiction with the opening angle of the GRB outflow. Cheng et al. (2011) pointed out that the angle is not the opening angle of the GRB outflow, but is just the collimation angle of the radiation of the corresponding string segment. We must caution that the existence of cosmic string is only speculative.

\section{Probing the Pop III stars and High-Redshift IGM}

\subsection{Observational signature of Pop III GRBs}

The first stars, also called Population III (Pop III) stars, are predicted to have formed in minihaloes with virial temperatures $T_{\text {vir }} \leq 10^{4} \mathrm{~K}$ at $z \geq 15$ (Tegmark et al. 1997; Yoshida et al. 2003; Bromm and Larson 2004). Numerical simulations show that Pop III stars forming in primordial minihaloes, were predominantly very massive stars with typical masses $M_{*} \geq$ $100 M_{\odot}$ (Bromm et al. 1999; Bromm and Loeb 2002; ; 2002), for recent reviews, see Bromm et al. (2009) and Bromm (2013). They had likely played a crucial role in early universe evolution, including reionization, metal enrichment history. Some studies shows that some Pop III stars will end as GRBs, called Pop III GRBs (Heger et al. 2003; Bromm and Loeb 2006; Komissarov and Barkov 2010; Stacy et al. 2011), which will be brighter and more energetic than any GRB yet detected (Toma et al. 2011; Nagakura et al. 2011; Campisi et al. 2011; Mészáros and Rees 2010; Nakauchi et al. 2012). Direct observations of the Pop III stars have so far been out of reach. The 

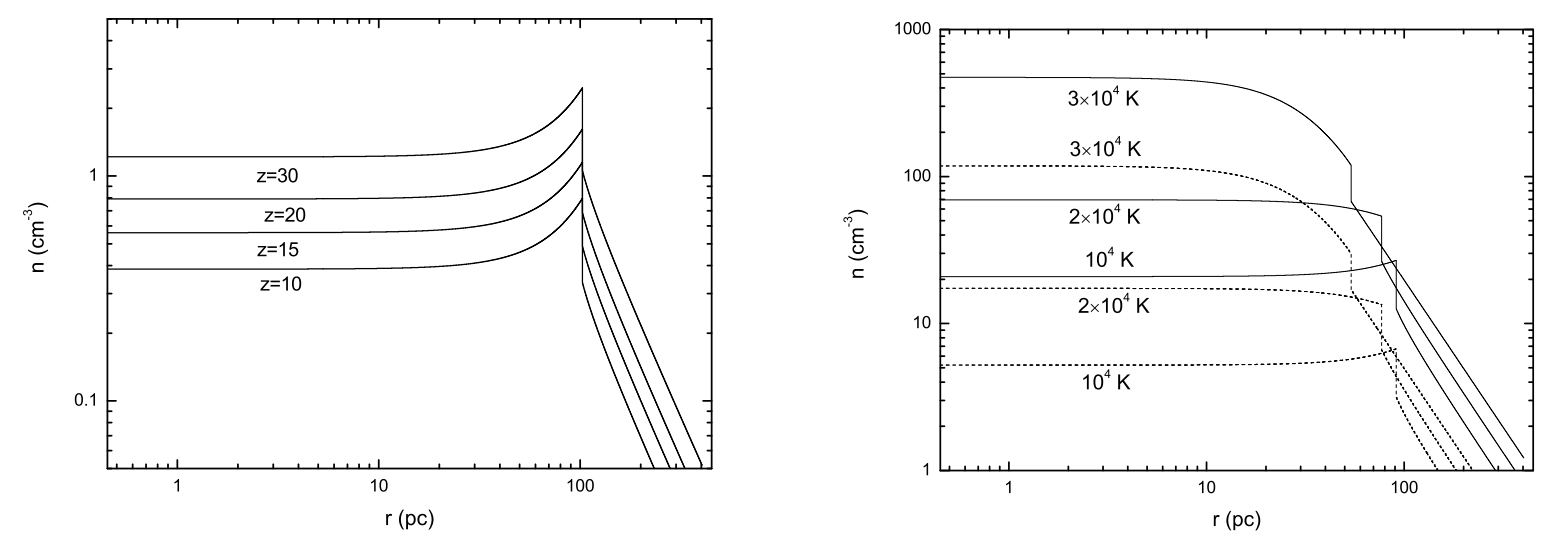

Figure 17: Left panel Minihalo circumburst density. Shown is the hydrogen number density as a function of distance from the central Pop III star at the moment of its death. Typical circumburst densities are $\sim 1 \mathrm{~cm}^{-3}$. Right panel Atomic-cooling-halo circumburst density. The density profiles are calculated from the Shu solution. The case of photoheating from only a single Pop III star (solid lines), and that from a stellar cluster (dotted lines). (Adapted from Figures 1 and 2 in Wang et al. (2012).)

properties of Pop III stars may be revealed by their remanents, Pop III GRBs.

In order to predict the observational signature of Pop III GRBs, the unusual circumburst environment that hosted the Pop III stars should be determined. In particular, the properties of the afterglow emission of Pop III GRBs depend on the circumburst density (Ciardi and Loeb 2000; Gou et al. 2004; Wang et al. 2012). In particular, the central minihalo environments just before a massive star die and a GRB bursts out can be understood as follows. The number of ionizing photons depends strongly on the central stellar mass, which is determined by a accretion flow onto the growing protostar (e.g., McKee and Tan 2008; Hosokawa et al. 2011; Stacy et al. 2012). Meanwhile the accretion is also affected by this radiation field. So the assembly of the Pop III stars and the development of an H II region around them proceed simultaneously, and affect each other. The shallow potential wells of minihalos are unable to maintain photo-ionized gas, so that the gas is effectively blown out of the minihalo. The resulting photoevaporation has been studied Alvarez et al. 2006; Abel et al. 2007; Greif et al. 2009).

The photoevaporation from minihalos can be described as the self-similar solution for a champagne flow (Shu et al. 2002). Assuming a $\rho \propto r^{-2}$ density profile, the spherically symmetric continuity and Euler equations for isothermal gas can be described as follows:

$$
\begin{gathered}
{\left[(v-x)^{2}-1\right] \frac{1}{\alpha} \frac{d \alpha}{d x}=\left[\alpha-\frac{2}{x}(x-v)\right](x-v),} \\
{\left[(v-x)^{2}-1\right] \frac{d v}{d x}=\left[(x-v) \alpha-\frac{2}{x}\right](x-v),}
\end{gathered}
$$

where $x=r / c_{s} t$, and $\rho(r, t)=\alpha(x) / 4 \pi G t^{2}=m_{\mathrm{H}} n(r) / X$ and $u(r, t)=c_{s} v(x)$ are the reduced density and velocity, respectively. $c_{s}$ is the sound speed and $X=0.75$ the hydrogen mass fraction. We set the typical lifetime of a massive Pop III star as $t=t_{*} \simeq 3 \times 10^{6} \mathrm{yr}$.

In the left panel of Figure 17 we show the density profiles at the end of the Pop III progenitor's life in the minihalo case. The circumstellar densities are nearly uniform at small radii. Such a flat density profile is markedly different from that created by stellar winds. But in the atomic cooling halo case, star formation and radiative feedback is not well understood (Johnson et al. 2009; Safranek-Shrader et al. 2012), such as the masses of stars, and stellar multiplicity (Clark et al. 2011). So we also use the formalism of the Shu solution as above. We assume that either one Pop III star or a small stellar cluster forms. The densities are shown in the right panel of Figure 17 Similar to the minihalo case, densities are nearly constant at small radii, but overall values are much higher, which is due to the deep potential wells, so that photoheated gas can easily be retained. Typical circumburst densities are $n \sim 100 \mathrm{~cm}^{-3}$. Pop III GRBs originating in atomic cooling halos may be extremely bright.

The typical parameters of the afterglow emission are adopted, $\Gamma_{0}=300, E_{\text {iso }}=10^{53} \mathrm{erg}, \Delta_{0}=10^{12} \mathrm{~cm}, \epsilon_{e}=0.3$, $\epsilon_{B}=0.1$, and $p=2.5$. As an example, in Figure 18, the M-band $\left(v=6.3 \times 10^{13} \mathrm{~Hz}\right)$ light curve is shown. Figure 19 gives the observed flux at $v=1.36 \times 10^{14} \mathrm{~Hz}$ as a function of redshift in the minihalo case. The lines with filled dots, black triangles and open dots correspond to an observed time of 6 minutes, 1 hour, and 1 day respectively. The straight line marks the K-band sensitivity for the near-infrared spectrograph (NIRSpec) on James Webb Space Telescope (Gardner et al. 2006). The high-redshift cut-off is due to the Ly $\alpha$ absorption. The flux will be completely absorbed by the intervening neutral IGM. At these frequencies, the flux of afterglow is weakly dependent on redshift of GRB. There are two reasons. First, the time dilation effect implies that the high redshift means the earlier emission times, where the afterglow are much brighter Ciardi and Loeb 2000; Bromm and Loeb 2012). Second, circumburst densities 


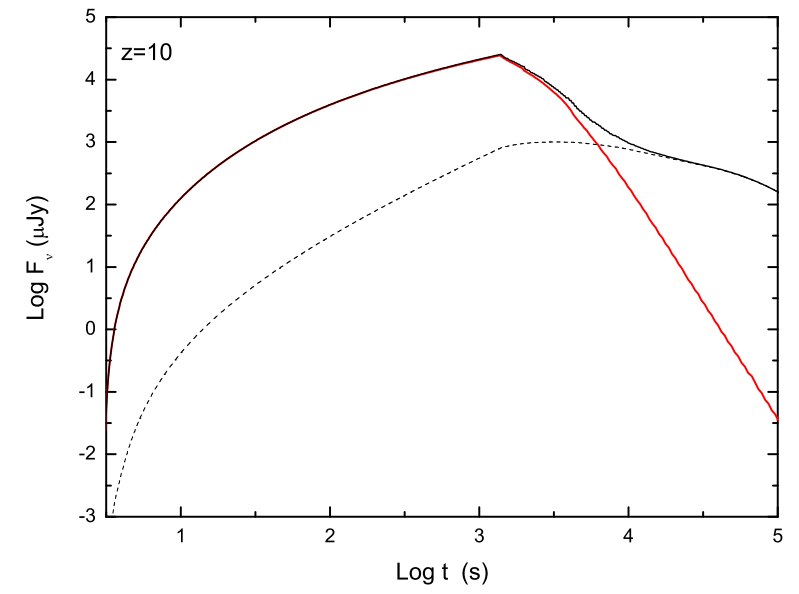

Figure 18: Light curve at $v=6.3 \times 10^{13} \mathrm{~Hz}$ (M band) of Pop III GRBs. The emission from the forward shock (dashed line), the reverse shock (solid red line), and their combination (solid black line) are shown. (Adapted from Figure 5 in Wang et al. (2012).)

of GRBs modestly increase with redshift.

\subsection{Metal enrichment history}

The metal enrichment history has several important influences for cosmic structure formation. For example, the metal injection change the mode of star formation (Bromm et al. 2001; Schneider et al. 2002). The transition between Pop III star formation and "normal" (Pop I/II) star formation has important implications, e.g., the expected GRB redshift distribution (Bromm and Loeb 2002), reionization (Wyithe and Loeb 2003), and the chemical abundance patterns of stars. So it is important to map the topology of pre-galactic metal enrichment. Ten or thirty meter-class telescopes have been proposed to measure the $z>6$ IGM metallicity with the GRB afterglow (Oh 2002). Meanwhile, the relative gas column density from metal absorption lines can reflect the enrichment history (Hartmann 2008; Wang et al. 2012).

Absorption processes and absorption lines imprinted on the spectra of GRBs or quasars are the main sources of information about the chemical and physical properties of high-redshift universe. But the bright QSO number is very low at $z>6$ (Fan et al. 2006). Meanwhile, there are several high-redshift GRBs: GRB 050904 at $z=6.29$, GRB 080913 at $z=6.7$, GRB 090423 at $z=8.3$ and GRB 090429B at $z=9.4$. The progenitors of long GRBs are thought to be massive stars, so the number of high-redshift GRBs does not decrease significantly. The density, temperature, kinematics and chemical abundances can be extracted from absorption lines (Oh 2002; Furlanetto and Loeb 2003). For instance, Kawai et al. (2006) have identified several metal absorption lines in the afterglow spectrum of GRB 050904 and found that this GRB occur in metal-enriched regions. Two absorption lines have been observed in the spectrum of GRB 090423 at $z=8.2$

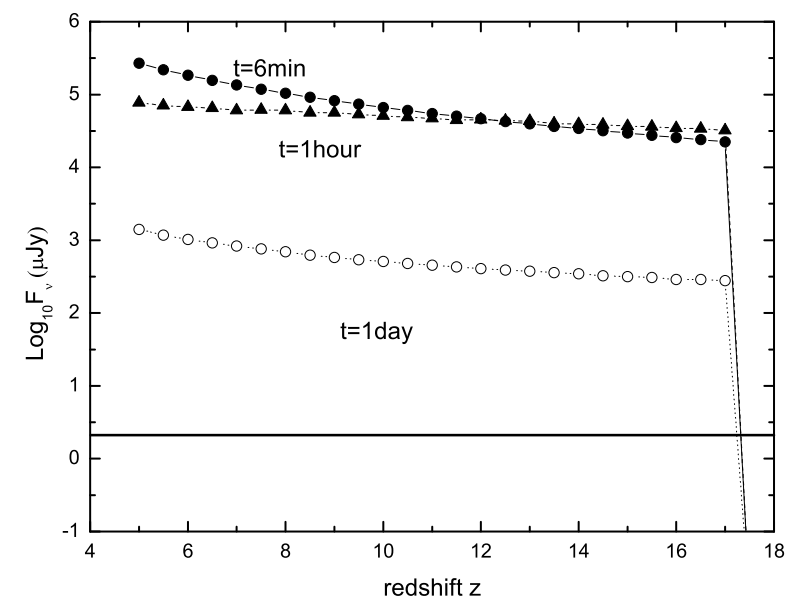

Figure 19: Observed flux at $v=1.36 \times 10^{14} \mathrm{~Hz}(\mathrm{~K}$ band) as a function of redshift at different observed times, as labelled. The K-band sensitivity of the NIRSpec instrument on board the JWST is shown as a horizontal line. The sharp cutoff at $z \simeq 17$ is due to Ly $\alpha$ absorption in the IGM. (Adapted from Figure 6 in Wang et al. (2012).)

(Salvaterra et al. 2009). These lines are due mainly to absorption metal elements in low ionization stages.

Wang et al. (2012) studied the ability of metal absorption lines in the spectra of Pop III GRBs to probe the pre-galactic metal enrichment. The first galaxy simulation carried out by Greif et al. (2010) was used. The simulation allowed one Pop III progenitor star to explode as an energetic supernova, then the IGM was polluted by the ejected metals. The simulation box size is $1 \mathrm{Mpc}$ (comoving), and is initialized at $z=99$ according to the $\Lambda$ CDM model with parameters: $\Omega_{\mathrm{m}}=1-\Omega_{\Lambda}=$ $0.3, \Omega_{\mathrm{b}}=0.04, h=H_{0} /\left(100 \mathrm{~km} \mathrm{~s}^{-1} \mathrm{Mpc}^{-1}\right)=0.7$, spectral in$\operatorname{dex} n_{\mathrm{s}}=1.0$, and normalization $\sigma_{8}=0.9$ (Spergel et al. 2003).

In Figure 20, the hydrogen number density and metallicity averaged along the line of sight are shown within the central $\simeq$ $100 \mathrm{kpc}$ closer to the virialization of the first galaxy at $z=16.4$. The distribution of metals produced by the first $\mathrm{SN}$ explosion is highly inhomogeneous, and the metallicity can reach up to $Z \sim$ $10^{-2.5} Z_{\odot}$, which is already larger than the critical metallicity, $Z_{\text {crit }} \leq 10^{-4} Z_{\odot}$. Therefore, both Pop III and Pop I/II stars will form during the assembly of the first galaxies (Johnson et al. 2008; Maio et al. 2010), so simultaneous occurrence of Pop III and normal GRBs at a given redshift (Bromm and Loeb 2006; de Souza et al. 2011). We consider a Pop III burst exploding in one of the (still metal-free) first galaxy progenitor minihalos at $z \simeq 16.4$.

For simplicity, we consider that prior to the GRB only one nearby SN exploded beforehand, dispersing its heavy elements into the pristine IGM. Two nucleosynthetic metal yields for Type II core-collapse SNe (Woosley and Weaver 1995), and for pair-instability supernovae (PISNe; Heger and Woosley 2002, 2010) are considered. Because the hydrogen is substantially neutral, metals will reside in states typical of C II, O I, Si II, 

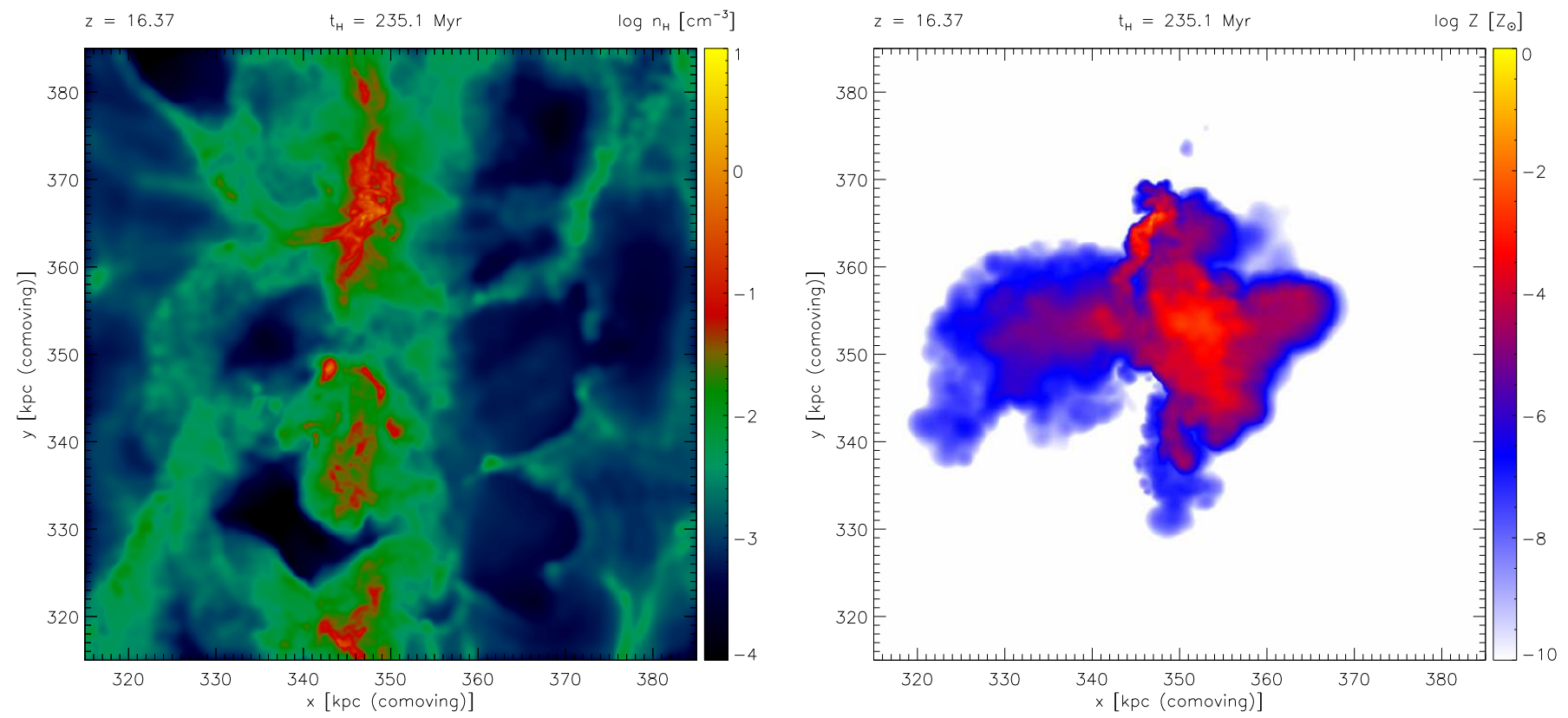

Figure 20: Possible explosion sites for high-redshift GRBs. Shown are the hydrogen number density (left panel) and metallicity contours (right panel) averaged along the line of sight at $z \sim 16.37$, when the first galaxy forms. The topology of metal enrichment is highly inhomogeneous. (Adapted from Figure 3 in Wang et al. (2012).)

and Fe II, because high-energy photons able to further ionize these elements will be absorbed by H I (Furlanetto and Loeb 2003).

Figure 21 shows two spectra of afterglow at the reverse shock crossing time. Top panel is for the top-heavy (Very Massive Star) initial mass function (PISN case) and bottom for normal initial mass function (Type II SNe case). The cutoff is due to Lyman- $\alpha$ absorption in the IGM which is expected to be still completely neutral at $z>10$. In the two cases, the metal lines are markedly different. The metal yields could be obtained from metal lines. So the initial mass function of Pop III stars can be derived from the metal absorption lines.

Strong absorption lines detected in GRB spectra, called damped Lyman- $\alpha$ (DLA) systems, could be used to probe the metal rnrichment (Savaglio 2006). For example, Castro-Tirado et al. (2013) detected the DLA systems of GRB $130606 \mathrm{~A}$ at $z=5.91$. The metallicity of this GRB environment is in the range from $\sim 1 / 7$ to $1 / 60$ of solar from the analysis of metal absorption lines. Figure 22 shows the metallicities derived from GRB-DLAs and QSO-DLAs. The GRB130606A sub-DLA is only the third GRB absorber with sub-DLA HI column density. So GRB sub-DLA is rare and hard to find. Simcoe et al. (2012) discovered the DLA of ULAS J1120+0641 at $z \sim 7$ with low metallicity. So GRB events at $z>10$ from future mission, offer an exciting new window to probe pre-galactic metal enrichment in high-redshift galaxies.

\subsection{Cosmic reionization}

The Gunn-Peterson (GP) test tells us that the IGM is almost fully ionized at $z \leq 5$ (Gunn and Peterson 1965). Reionization of the IGM is thought to have occurred during $z \sim 6-20$ by

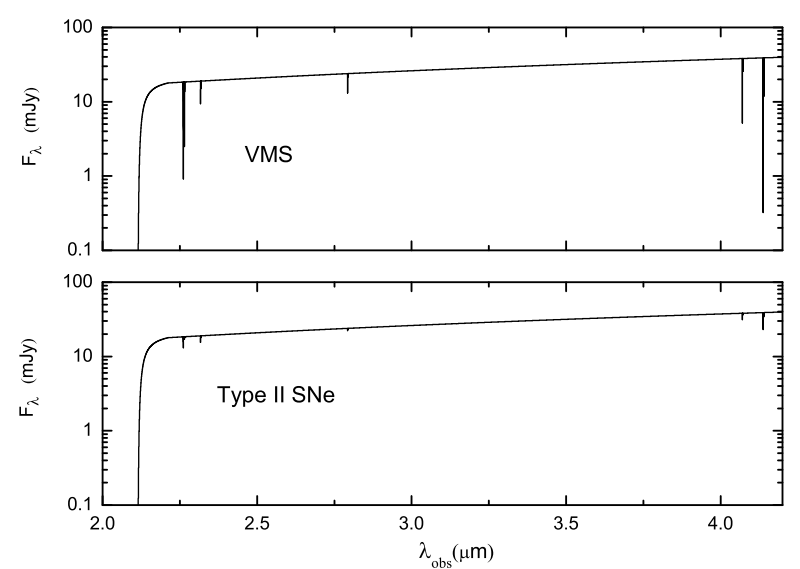

Figure 21: Pop III GRB spectrum observed at the reverse shock crossing time $t_{\oplus}=16.7 \times(1+16.4) \mathrm{s}$. Metal absorption lines are imprinted according to the Pop III SN event, PISN vs. core-collapse. The former originates from a very massive star (VMS) progenitor, whereas the latter from a less massive one. In each case, the cutoff at short wavelengths is due to Lyman- $\alpha$ scattering in the neutral IGM. Adapted from Figure 10 in Wang et al. (2012).) 


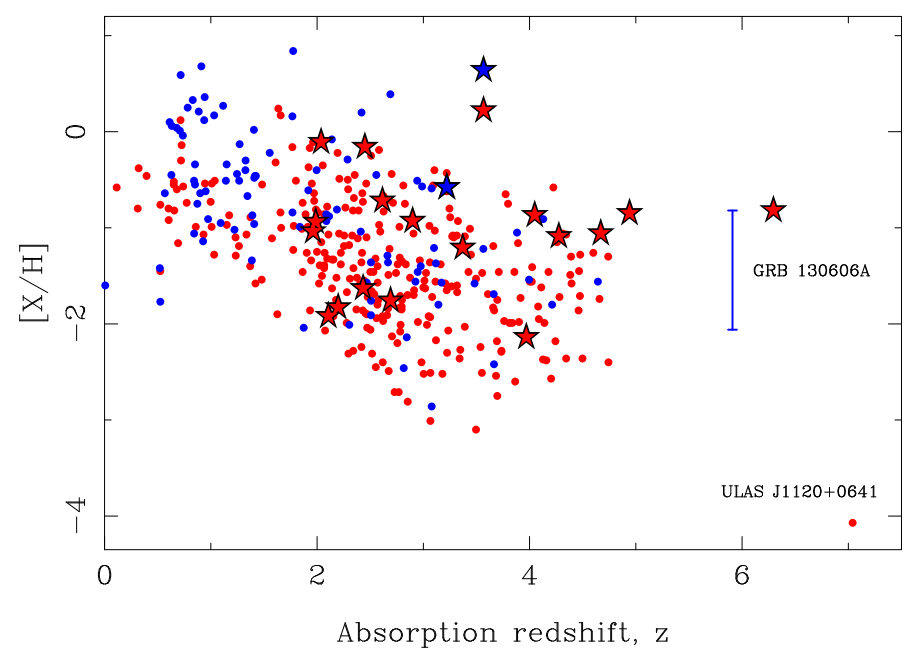

Figure 22: The metallicity $([\mathrm{X} / \mathrm{H}])$ as a function of redshift is shown for QSO-DLAs (circles) and GRB-DLAs (stars Schady et al. 2011; Thöne et al. 2013), including GRB 130606A at $z=5.91$ and ULAS J1120+0641 at z 7 (Simcoe et al. 2012). Blue colors are derived for $\log \mathrm{N}(\mathrm{HI})<20.3$ and red is derived for $\log \mathrm{N}(\mathrm{H} \mathrm{I}) \geq 20.3$. (Adapted from Figure 8 in Castro-Tirado et al. (2013).

Pop III stars and/or quasars, and the precise measurement of the reionization is one of the key topics in modern cosmology (for reviews, see Barkana and Loeb 2001; Robertson et al. 2010). The absorption of GRB afterglow is dependent on the structure of reionization and the global history of reionization, so it has the potential to distinguish between different theoretical models of reionization (Miralda-Escude 1998; McOuinn et al. 2008). The afterglows at wavelengths close to the Ly $\alpha$ resonance potentially provide a sensitive probe of the ionization fraction in the IGM (Miralda-Escude 1998; Barkana and Loeb 2004). The IGM neutral fraction could be derived by fitting red damping wing with high precision. The absorption from host galaxy complicates the measurement of the IGM ionization state from a GRB spectrum, but in principle this absorption is less extended in wavelength and could be separated. Some studies show that $20-30 \%$ of GRB host galaxies have small HI column density to allow determination of the absorption from a partially ionized IGM (Chen et al. 2007).

By studying the afterglow spectrum of GRB 050904 at $z=$ 6.3, Totani et al. (2006) found that the IGM was already largely ionized at $z=6.3$, and the upper limit of 0.17 for the neutral fraction of IGM at $68 \%$ confidence level. But the absorption from host galaxy dominates the absorption redward of the Ly $\alpha$ forest, which limits the constraints on reionization (Totani et al. 2006; McQuinn et al. 2008). The bright optical afterglow of GRB $130606 \mathrm{~A}$ at $z=5.9$ gives an opportunity to probe the ionization status of IGM. The neutral fraction of IGM is found to be 0.1 to 0.5 by analyzing of the red $\operatorname{Ly} \alpha$ damping wing of the afterglow spectrum taken by Subaru (Totani et al. 2013).

From theoretical view, the reionization process can also be studied through theoretical model. The average evolution of $Q_{\mathrm{HII}}=n_{e} / n_{H}$ is derived by numerical integration of the rate of ionizing photons minus the rate of radiative recombinations (Madau et al. 1999; Barkana and Loeb 2001; Wyithe and Loeb

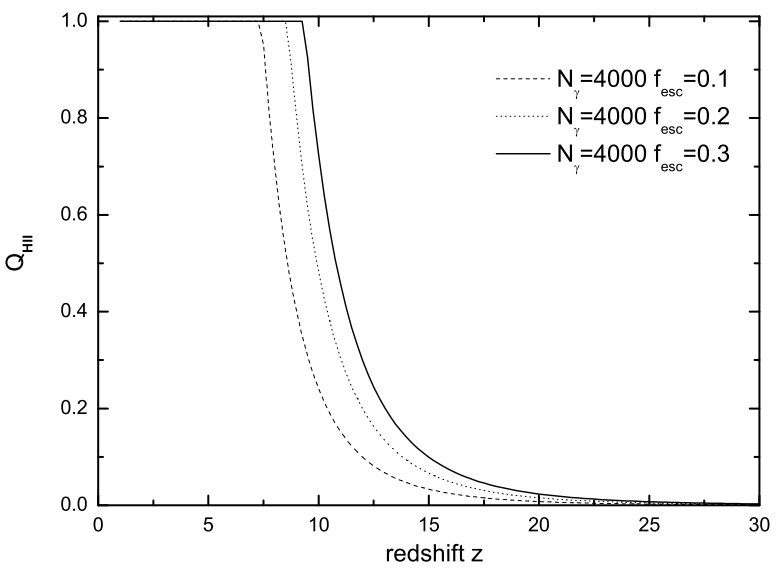

Figure 23: The HII filling factor $Q_{\mathrm{HII}}$ as a function of redshift computed for different values of $f_{\text {esc }}$. (Adapted from Figure 5 in Wang (2013).)

2003; Yu et al. 2012)

$$
\frac{d Q_{\mathrm{HII}}}{d z}=\left(\frac{\dot{N}_{\text {ion }}}{n_{H}}-\alpha_{B} C n_{H} Q_{\mathrm{HII}}\right) \frac{d t}{d z},
$$

where

$$
\dot{N}_{\text {ion }}=(1+z)^{3} \dot{\rho}_{*}(z) N_{\gamma} f_{\text {esc }} / m_{p}
$$

is the rate of ionizing photons ejected into the IGM, $N_{\gamma}$ is the number of ionizing photons, $\dot{\rho}_{*}(z)$ is the SFR and $f_{\text {esc }}$ is the escape fraction. Using the SFR derived from GRBs in section 3.1 the evolution of the HII volume filling factor $Q_{\mathrm{HII}}$ can be numerically calculated from equation (39). Figure 23 shows the evolution of $Q_{\mathrm{HII}}$ as a function of redshift.

The cosmic microwave background (CMB) optical depth back to redshift $z$ is also seriously depend on the reionization history, which can be written as the integral of $n_{e} \sigma_{T} d \ell$, i.e.,

$$
\tau_{e}(z)=\int_{0}^{z} n_{e}(z) \sigma_{T}\left(1+z^{\prime}\right)^{-1}\left[c / H\left(z^{\prime}\right)\right] d z^{\prime} .
$$

The optical depth is shown in Figure 24. The WMAP nine-year data gives $\tau_{e}=0.089 \pm 0.014$ (Hinshaw et al. 2013), which is shown as the shaded region. The combination of Planck and WMAP data also gives $\tau_{e}=0.089_{-0.014}^{+0.012}$ (Planck Collaboration 2014). So GRB-inferred SFR can reproduce the CMB optical depth. But the value of the escape fraction $f_{\text {esc }}$ (Robertson et al. 2010) and clumping factor $C$ are hard to determined.

\section{Summary and future prospect}

GRBs are observed throughout the whole electromagnetic spectrum, from radio waves to $\gamma$-rays, which have been observed in distant universe. Recently, GRBs have attracted a lot of attention as promising standardizable candles to construct the Hubble diagram to high redshift, as complementarity to other cosmological probes, such as SNe Ia, CMB and BAO. However, a lot of work is needed to be sure that GRBs can hold 


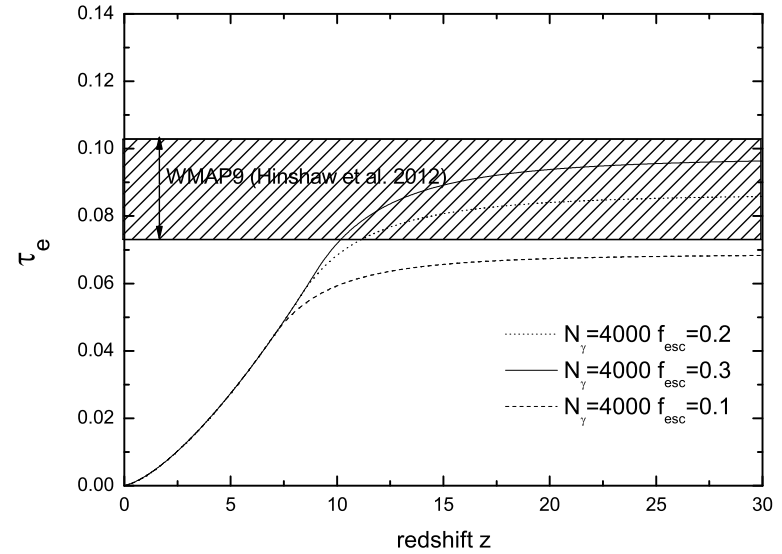

Figure 24: The optical depth $\tau_{e}$ due to the scattering between the ionized gas and the CMB photons is shown. The shade region is given by the nine-year WMAP measurements. The reionization history calculated from GRB-inferred SFR can easily reach $\tau_{e}$ from WMAP nine-year data. Adapted from Figure 6 in Wang (2013).)

this promise in future. The most important thing is to search for a correlation similar to that used to standardize SNe Ia. In order to obtain the correlation, the classification of GRBs may be crucial. We must remind that only SNe Ia are standard candles among all SNe. The classical classification method is based on the prompt emission properties (duration, hardness, and spectral lag). The physics of prompt emission are not fully understood (Zhang 2014), and some new clues from other objects are found (Wang and Dai 2013; Wang et al. 2014). But observations of some GRBs are challenging the standard classification (Zhang 2006; Zhang et al. 2009; Lü et al. 2010). So more physical nature of GRBs is needed (Zhang et al. 2009). The circularity problem could be partially solved by analyzing a sample of GRBs within a small redshift bin (Lamb et al. 2005; Ghirlanda et al. 2006a; Liang and Zhang 2006). In particular, Liang and Zhang (2006) found that one can calibrate the power law indices of various standard candle correlations with this method.

In order to measure high-redshift SFR from GRBs, the relation between long GRB rate and SFR must be known. Besides, theoretical models of the SFR have several free parameters, such as the efficiency of star formation and the chemical feedback strength. From the theoretical SFR, the predicted GRB redshift distribution can be derived. So one can use the GRB redshift distribution observed by Swift (or future missions such as SVOM and EXIST), to calibrate the free parameters. More GRB red damping wing with low HI column density are required to study properties of IGM.

Metal absorption lines in the GRB afterglow spectrum, giving rise to EWs of a few tens of $\AA$, which may allow us to distinguish whether the first heavy elements were produced in a Pop III star died as a PISN or a core-collapse SN. To this extent, the spectrum needs to be obtained sufficiently early, within the first few hours after the trigger. Upcoming JWST would detect much more high-redshift GRBs (properly Pop III GRBs) with high resolution NIR spectra including metal absorption lines, which allow one to measure the cosmic metallicity evolution.

In the future, the French-Chinese satellite Space-based multiband astronomical Variable Objects Monitor (SVOM) and JWST, have been optimized to increase the number of GRB and the synergy with the ground-based facilities. There are a combination of multi-wavelength detectors on board of SVOM (Paul et al. 2011). ECLAIRs wide-field camera will detect GRBs in the energy range of $4-150 \mathrm{keV}$. The spectral information of prompt emission will be measured by Gamma-Ray Monitor (GRM). The afterglow can be obtained by the Micro channel X-ray Telescope (MXT; 0.3-10 keV) and the Visible Telescope (VT; 400-900nm). SVOM can detect about 80 GRBs per year, and more than $50 \%$ of GRBs have redshift measurement (Petitjean and Vergani 2011). JWST is a large, infraredoptimized space telescope with $6.6 \mathrm{~m}$ diameter aperture. It has four scientific instruments: a Near-IR Camera (NIRCam), a Near-IR Spectrograph (NIRSpec), a near-IR Tunable Filter Imager (TFI), and a Mid-IR Instrument (MIRI) (Gardner et al. 2006). But the direct detection of a single Pop III star is not feasible even for JWST, i.e., the AB magnitude of a $M=1000 M_{\odot}$ star is only 36 at $z \sim 30$. Meanwhile, the Pop III GRBs can be detectable by JWST (Wang et al. 2012; Mesler et al. 2014; Macpherson et al. 2013). This will boost the amount of information available to tackle the important issues revealed by this exciting field of research.

\section{Acknowledgements}

We thank the anonymous referee for detailed and very constructive suggestions that have allowed us to improve our manuscript. We thank Shuang-Nan Zhang for helpful discussions and comments. This work is supported by the National Basic Research Program of China (973 Program, grant No. 2014CB845800), the National Natural Science Foundation of China (grants 11422325, 11373022, and 11033002), the Excellent Youth Foundation of Jiangsu Province (BK20140016), and the Program for New Century Excellent Talents in University (grant No. NCET-13-0279).

\section{References}

Abel, T., Bryan, G. L., Norman, M. L. 2002, Science, 295, 93

Abel, T., Wise, J. H., Bryan, G. L. 2007, ApJ, 659, L87

Allen, S. W., et al. 2004, MNRAS, 353, 457

Alvarez, M. A., Bromm, V., Shapiro, P. R. 2006, ApJ, 639, 621

Amati, L., 2006, MNRAS, 372, 233

Amati, L., Della Valle, M., 2013, Int. J. Mod. Phys. D, 22, 1330028

Amati, L. et al., 2002, A\&A, 390, 81

Amati, L., et al., 2008, MNRAS, 391, 577

Amati, L., Frontera, F., Guidorzi, C., 2009, A\&A, 508, 173

Band, D. et al., 1993, ApJ, 413, 281

Band, D., Norris, J., Bonnell, J., 2004, ApJ, 613, 484

Band, D. L., Preece, R. D., 2005, ApJ, 627, 319

Bannister, K. W., Murphy, T., Gaensler, B. M., Reynolds, J. E., 2012, ApJ, 757, 38

Barkana, R., Loeb, A. 2001, Phys. Rep., 349, 125

Barkana, R., Loeb, A., 2004, ApJ, 601, 64 
Basilakos, S., Perivolaropoulos, L., 2008, MNRAS, 391, 411

Bernardini, M. G., et al., 2015, MNRAS, 446, 1129

Bertolami, O., Silva, P. T., 2006, MNRAS, 356, 1149

Bloom, J. S., Frail, D. A., Kulkarni, S. R., 2003, ApJ, 594, 674

Bloom, J. S., Frail, D. A., Sari, R., 2001, AJ, 112, 2879

Bond, J. R., Efstathiou, G., 1984, ApJ, 285, L45

Bond, J. R., Efstathiou, G., Tegmark, M., 1997, MNRAS, 294, L33

Bouwens, R. J., Illingworth, G. D., Franx, M., et al. 2009, ApJ, 705, 936

Bouwens, R. J., Illingworth, G. D., Labbe, I., et al. 2011, Nature, 469, 504

Bromm, V., 2013, Rep. Prog. Phys., 76, 2901

Bromm, V., Coppi, P. S., Larson, R. B., 1999, ApJ, 527, L5

Bromm, V., Coppi, P. S., Larson, R. B., 2002, ApJ, 564, 23

Bromm, V., Ferrara, A., Coppi, P. S., Larson, R. B., 2001, MNRAS, 328, 969

Bromm, V., Larson, R. B., 2004, ARA\&A, 42, 79

Bromm, V., Loeb, A., 2002, ApJ, 575, 111

Bromm, V., Loeb, A., 2006, ApJ, 642, 382

Bromm, V., Loeb, A., 2012, in Gamma-ray Bursts, ed. C. Kouveliotou, S

E. Woosley, R. A. M. J. Wijers (Cambridge: Cambridge Univ. Press), arXiv:0706.2445 2

Bromm, V., Yoshida, N., Hernquist, L., McKee, C. F., 2009, Nature, 459, 49

Butler, N. R., Kocevski, D., Curtis, J. L., Bloom, J. S., 2007, ApJ, 671, 656

Campana, S., Guidorzi, C., Tagliaferri, G., Chincarini, G., Moretti, A., Rizzuto,

D., Romano, P., 2007, A\&A, 472, 395

Campisi, M. A., Li, L. X., Jakobsson, P., 2010, MNRAS, 407, 1972

Campisi, M. A., Maio, U., Salvaterra, R., Ciardi, B., 2011, MNRAS, 416, 2760

Cao, X. F., Yu, Y. W., Cheng, K. S., Zheng, X. P., 2011, MNRAS, 416, 2174

Capozziello, S., et al., arXiv:1206.6700

Capozziello, S., Izzo, L., 2008, A\&A, 490, 31

Cardone, V. F., Capozziello, S., Dainotti, M. G., 2009, MNRAS, 400, 775

Cardone, V. F., Dainotti, M. G., Capozziello, S., Willingale, R., 2010, MNRAS, 408,1181

Castro-Tirado, A. J., Sánchez-Ramírez, R., Ellison, S. L., et al., 2013, arXiv:1312.5631

Cattoën, C., Visser, M., preprint, gr-qc/0703122 3

Cen, R., 2003, ApJ, 591, 12

Chen, H. W., Prochaska, J. X., Gnedin, N. Y., 2007, ApJ, 667, L125

Cheng, K. S., Yu, Y., Harko, T., 2010, Phys. Rev. Lett., 104, 241102

Cheng, K. S., Yu, Y., Harko, T., 2010, Phys. Rev. Lett., 106, 259002

Ciardi, B., Loeb, A., 2000, ApJ, 540, 687

Clark, P. C., Glover, S. C. O., Klessen, R. S., Bromm, V., 2011, ApJ, 727, 110

Coward, D., 2007, New Astronomy Reviews, 51, 539

Crider, A., Liang, E. P., Preece, R. D., et al., 1999, ApJ, 519, 206

D'Agostini, G., 2005, preprint (arXiv: physics/0511182)

Dai, Z. G., Liang, E. W., Xu, D., 2004, ApJ, 612, L101

Dai, Z. G., Wang, F. Y., 2007, Advances in Space Research, 40, 1244

Daigne, F., Rossi, E. M., Mochkovitch, R., 2006, MNRAS, 372, 1034

Dainotti, M. G., Cardone, V. F., Capozziello, S., 2008, MNRAS, 391, L79

Dainotti, M. G., et al., 2014, arXiv: 1412.3969

Davé, R., 2008, MNRAS, 385, 147

Demianski, M., Piedipalumbo, E., Rubano, C., 2011, MNRAS, 411, 1213

Demianski, M., Piedipalumbo, E., 2011, MNRAS, 415, 3580

Deng, W., Zhang, B., 2014, ApJL, 783, 35

de Souza, R. S., Yoshida, N., Ioka, K., 2011, A\&A, 533, A32

de Souza, R. S., et al., 2013, MNRAS, 432, 3218

Di Girolamo, T., et al., 2005, J. Cosmol. Astropart. Phys., 4, 8

Eichler, D., Levinson, A., 2004, ApJ, 614, L13

Eichler, D., Levinson, A., 2006, ApJ, 649, L5

Eisenstein, D. J., et al. 2005, ApJ, 633, 560

Elliott, J., Greiner, J., Khochfar, S., et al. 2012, A\&A, 539, A113

Efron, B., Petrosian, V., 1992, ApJ, 399, 345

Fan, X., Carilli, C. L., Keating, B., 2006, ARA\&A, 44, 415

Fenimore, E. E., Ramirez-Ruiz, E., 2000, preprint (astro-ph/0004176)

Firmani, C., Ghisellini, G., Ghirlanda, G., Avila-Reese, V., 2005, MNRAS, 360, L1

Firmani, C., Avila-Reese, V., Ghisellini, G., Ghirlanda, G., 2006, MNRAS, 370,185

Firmani, C., Cabrera, J. I., Avila-Reese, V., 2009, MNRAS, 393, 1209

Frail, D. A., et al., 2001, ApJL, 562, 55

Frebel, A., Johnson, J. L., Bromm, V., 2007, MNRAS, 380, L40

Frebel, A., Johnson, J. L., Bromm, V., 2009, MNRAS, 392, L50

Friedman, A. S., Bloom, J. S., 2005, ApJ, 627, 1
Frontera, F., Amati, L., Guidorzi, C., 2012, ApJ, 754, 138

Fruchter, A. S., Levan, A. J., Strolger, L., et al. 2006, Nature, 441, 463

Fruchter, A. S., Thorsett, S. E., Metzger, M. R., et al., 1999, ApJL, 519, 13

Furlanetto, S. R., Loeb, A., 2003, ApJ, 588, 18

Furlanetto, S. R., Loeb, A., 2005, ApJ, 634, 1

Fynbo, J. P. U., Malesani, D., Jakobsson, P., 2012, Gamma-Ray Bursts, Cambridge Astrophysics Series 51, 269

Gao, H., Li, Z., Zhang, B., 2014, ApJ, 788, 189

Gao, H., Liang, N., Zhu, Z. H., 2012, Int. J. Mod. Phys. D, 21, 1250016

Gardner, J. P.,Mather, J. C., Clampin, M., et al., 2006, Space Sci. Rev., 123, 485

Gehrels, N., 1986, ApJ, 303, 336

Gehrels, N. et al., 2004, ApJ, 611, 1005

Gehrels, N. et al., 2006, Nature, 444, 1044

Gehrels, N., Ramirez-Ruiz, E., Fox, D. B., 2009, ARA\&A, 47, 567

Ghirlanda, G., 2009, AIP Conf. Proc., 1111, 579

Ghirlanda, G., Ghisellini, G., Firmani, C. 2006a, New J. Phys., 8, 123

Ghirlanda, G., Ghisellini, G., Firmani, C., Celotti, A., Bosnjak, Z., 2005, MNRAS, 360, L45

Ghirlanda, G., et al. 2006b, A\&A, 452, 839

Ghirlanda, G., Ghisellini, G., Lazzati, D. 2004a, ApJ, 616, 331

Ghirlanda, G., et al. 2004b, ApJ, 613, L13

Ghirlanda, G., et al. 2011, MNRAS, 418, L109

Ghirlanda, G., et al. 2012, MNRAS, 422, 2553

Ghirlanda, G., Nava, L., Ghisellini, G., 2010, A\&A, 511, A34

Ghirlanda, G., Nava, L., Ghisellini, G., Firmani, C., Cabrera, J. I., 2008, MNRAS, 387, 319

Graham, J. F., et al,. 2009, AIP Conf. Proc. Ser. 1133, 269

Greif, T. H., Glover, S. C. O., Bromm, V., Klessen, R. S., 2010, ApJ, 716, 510

Greif, T. H., Johnson, J. L., Klessen, R. S., Bromm, V., 2009, MNRAS, 399, 639

Gou, L. J., Mészáros, P., Abel, T., Zhang, B., 2004, ApJ, 604, 508

Guetta, D., Piran, T., 2007, J. Cosmol. Astropart. Phys., 7, 3

Guetta, D., Piran, T., Waxman, E., 2005, ApJ, 619, 412

Guidorzi, C., et al., 2006, MNRAS, 371, 843

Gunn, J. E., Peterson, B. A., 1965, ApJ, 142, 1633

Hao, J. M., Yuan, Y. F., 2013, ApJ, 772, 42

Hartmann, D. H., 2008, New Astronomy Reviews, 52, 450

Heger, A., et al., 2003, ApJ, 591, 288

Heger, A., Woosley, S. E., 2002, ApJ, 567, 532

Heger, A., Woosley, S. E., 2010, ApJ, 724, 341

Hinshaw, G., Larson, D., Komatsu, E., et al., 2013, ApJS, 208, 19

Hirschi, R., Meynet, G., Maeder, A., 2005, A\&A, 443, 581

Hjorth, J., Sollerman, J., Möller, P., et al., 2003, Nature, 423, 847

Holz, D. E., 1998, ApJ, 506, L1

Hooper, D., Dodelson, S., 2007, APh, 27, 113

Hopkins, A. M., Beacom, J. F., 2006, ApJ, 651, 142

Hosokawa, T., Omukai, K., Yoshida, N., Yorke, H. W., 2011, Science, 334, 1250

Hu, W., Sugiyama. N., 1996, ApJ, 471, 30

Ioka, K., Nakamura, T., 2001, ApJ, 554, L163

Johnson, J. L., Greif, T. H., Bromm, V., 2008, MNRAS, 388, 26

Johnson, J. L., Greif, T. H., Bromm, V., et al., 2009, MNRAS, 399, 37

Jimenez, R., Piran, T., 2013, ApJ, 773, 126

Jimenez, R., Verde, L., Treu, T., Stern, D., 2003, ApJ, 593, 622

Jones, D., et al., 2013, ApJ, 768, 166

Karlsson, T., Bromm, V., Bland-Hawthorn, J., 2012, Rev. Mod. Phys., 85, 809

Kawai, N., Kosugi, G., Aoki, K., et al., 2006, Nature, 440, 184

Kistler, M. D., et al., 2008, ApJ, 673, L119

Kistler, M. D., et al., 2009, ApJ, 705, L104

Kobayashi, S., Ryde, F., MacFadyen, A., 2002, ApJ, 577, 302

Kodama, Y., et al., 2008, MNRAS, 391, L1

Kocevski, D., West, A. A., Modjaz, M., 2009, ApJ, 702, 377

Komatsu, E., Smith, K. M., Dunkley, J., et al., 2011, ApJS, 192, 18

Komissarov, S. S., Barkov, M. V., 2010, MNRAS, 402, L25

Kouveliotou, C., et al., 1993, ApJ, 413, L101

Kudritzki, R. Puls, J., 2000, ARA\&A, 38, 613

Kumar, P., Zhang, B., 2014, arXiv:1410.0679

Lamb, D. Q., et al., 2005, astro-ph/0507362

Lamb, D. Q., Reichart, D. E., 2000, ApJ, 536, 1

Langer, L., Norman, C. A., 2006, ApJ, 638, L63 
Le, T., Dermer, C. D., 2007, ApJ, 661, 394

Le Floc'h, E. et al., 2003, A\&A, 400, 499

Levesque, E. M., 2014, PASP, 126, 1

Levesque, E. M., Kewley, L. J., Berger, E., Jabran, Zahid H., 2010, AJ, 140, 1557

Li, H., et al., 2008a, PLB, 658, 95

Li, H., et al., 2008b, ApJ, 680, 92

Li, L. X., 2007, MNRAS, 379, L55

Li, L. X. 2008, MNRAS, 388, 1487

Li, L. X., Paczyński, B., 2006, MNRAS, 366, 219

Liang, E. W., Dai, Z. G., Wu, X. F., 2004, ApJ, 606, L29

Liang, E. W. et al., 2006, ApJ, 653, L81

Liang, E. W., Zhang, B. 2005, ApJ, 633, 611

Liang, E. W., Zhang, B. 2006, MNRAS, 369, L37

Liang, N., Xiao, W. K., Liu, Y., Zhang, S. N., 2008, ApJ, 685, 354

Liang, N., Wu, P., Zhang, S. N., 2008, Phys. REv. D, 81, 083518

Lin, J. R., Zhang, S. N., Li, T. P., 2004, ApJ, 605, 819

Llyd-Ronning, N. M., Fryer, C. L., Ramirez-Ruiz, E. 2002, ApJ, 574, 554

Lü, H. J., Liang, E. W., Zhang, B. B., Zhang, B., 2010, ApJ, 725, 1965

Lynden-Bell, D., 1971, MNRAS, 155, 95

MacFadyen, A. I., Woosley, S. E. 1999, ApJ, 524, 262

Madau, P., Ferrara, A., Rees, M. J. 2001, ApJ, 555, 92

Madau, P., Haardt, F., Rees, M. J. 1999, ApJ, 514, 648

Macpherson, D., Coward, D. M., Zadnik, M. G., 2013, ApJ, 779, 73

Maio, U., Ciardi, B., Dolag, K., Tornatore, L., Khochfar, S. 2010, MNRAS, 407, 1003

Maio, U., et al., 2012, MNRAS, 426, 2078

McKee, C. F., Tan, J. C. 2008, ApJ, 681, 771

McQuinn, M., et al., 2008, MNRAS, 388, 1101

Mesinger, A., Perna, R., Haiman, Z., 2005, ApJ, 623, 1

Mesler, R. A., et al., 2014, ApJ, 787, 91

Mészáros, P., 2006, Rep. Prog. Phys., 69, 2259

Mészáros, P., Ramirez-Ruiz, E., Rees, M. J., Zhang, B., 2002, ApJ, 578, 812

Mészáros, P., Rees, M. J. 2010, ApJ, 715, 967

Miralda-Escude, J., 1998, ApJ, 501, 15

Mosquera Cuesta, H. J., Dumet M, H., Furlanetto, C., 2008, JCAP, 07, 004

Mörtsell, E., Sollerman, J., 2005, JCAP, 06, 009

Nagakura, H., et al., 2011, 731, 80

Nakar, E., Piran, T., 2005, MNRAS, 360, L73

Nakauchi, D., et al., 2012, ApJ, 759, 128

Natarajan, P., Albanna, B., Hjorth, J., et al., 2005, MNRAS, 364, L8

Nava, L., et al., 2006, A\&A, 450, 471

Nava, L., Ghirlanda, G., Ghisellini, G., Firmani, C., 2008, MNRAS, 391, 639

Niino, Y., 2011, MNRAS, 417, 567

Norris, J. P., Marani, G. F., Bonnell, J. T. 2000, ApJ, 534, 248

Oh, S. P. 2002, MNRAS, 336, 1021

Oppenheimer, B. D., Davé, R., Finlator, K. 2009, MNRAS, 396, 729

Oguri, M., Takahashi, K. 2006, Phys. Rev. D, 73, 123002

Pan, Y., et al., 2013, PLB, 718, 699

Paul, J., Wei, J. Y., Basa, S., Zhang, S. N., 2011, Comptes Rendus Physique, 12,198

Peebles, P. J. E., Yu, J. T. 1970, ApJ, 162, 815

Perlmutter, S., et al. 1999, ApJ, 517, 565

Petitjean, P., Vergani, S. D. 2011, Comptes Rendus Physique, 12, 288

Planck Collaboration, 2014, A\&A, 571, A16

Porciani, C., Madau, P., 2001, ApJ, 548, 522

Price, P. A., et al. 2007, ApJ, 663, L57

Prochaska, J. X., Chen, H.-W., Dessauges-Zavadsky, M., Bloom, J. S. 2007, ApJ, 666, 267

Qi, S., Lu, T., 2010, ApJ, 717, 1274

Qi, S., Lu, T., Wang, F. Y., 2009, MNRAS, 398, L78

Qi, S., Wang, F. Y., Lu, T., 2008a, A\&A, 483, 49

Qi, S., Wang, F. Y., Lu, T., 2008b, A\&A, 487, 853

Qian, Y. Z., Wasserburg, G. J. 2001, ApJ, 559, 925

Qin, S. F., Liang, E. W., Lu, R. J., Wei, J. Y., Zhang, S. N. 2010, MNRAS, 406, 558

Reichart, D. E., Lamb, D. Q., Fenimore, E. E., et al., 2001, ApJ, 552, 57

Rhoads, J. E., 1997, ApJL, 487, 1

Riess, A. G., et al. 1998, AJ, 116, 1009

Rizzuto, D. et al., 2007, MNRAS, 379, 619

Robertson, B. E., Ellis, R. S. 2012, ApJ, 744, 95
Robertson, B. E., et al., 2010, Nature, 468, 49

Safranek-Shrader, C., Agarwal, M., Federrath, C., et al. 2012, MNRAS, 426, 1159

Sakamoto, T., Barthelmy, S. D., Baumgartner, W. H., et al., 2011, ApJS, 195, 2

Salvaterra, R., Campana, S., Vergani, S. D., et al. 2012, ApJ, 749, 68

Salvaterra, R., Chincarini, G., 2007a, ApJ, 656, L49

Salvaterra, R., Della Valle, M., Campana, S., et al. 2009, Nature, 461, 1258

Sari, R., 1999, ApJL, 524, 43

Savaglio, S., 2006, NJPh, 8, 195

Savaglio, S. et al., 2005, ApJ, 635, 260

Schady, P., Savaglio, S., Kruhler, T., Greiner, J., Rau, A. 2011, A\&A, 525, A113

Schaefer, B. E. 2003, ApJ, 588, 387

Schaefer, B. E. 2007, ApJ, 660, 16

Schaefer, B. E., Deng, M., Band, D. L. 2001, ApJ, 563, L123

Schmidt, M., 1999, ApJ, 523, L117

Schneider, R., Ferrara, A., Natarajan, P., Omukai, K. 2002, ApJ, 571, 30

Schneider, R., Omukai, K., Inoue, A. K., Ferrara, A. 2006, MNRAS, 369, 1437

Shahmoradi, A., Nemiroff, R. J., 2011, MNRAS, 411, 1843

Shu, F. H., Lizano, S., Galli, D., Cantó, J., Laughlin, G., 2002, ApJ, 580, 969

Silk, J., 1968, ApJ, 151, 459

Simcoe, R. A., Sullivan, P. W., Cooksey, K. L., et al. 2012, Nature, 492, 79

Soderberg, A. M. et al., 2004, Nat, 430, 648

Sollerman, J., Östlin, G., Fynbo, J. P. U., Hjorth, J., Fruchter, A., Pedersen, K. 2005, New Astron., 11, 103

Spergel, D. N., et al. 2003, ApJS, 148, 175

Stacy, A., Bromm, V., Loeb, A. 2011, MNRAS, 413, 543

Stacy, A., Greif, T. H., Bromm, V. 2012, MNRAS, 422, 290

Stanek, K. Z., Matheson, T., Garnavich, P. M., et al. 2003, ApJ, 591, L17

Stanek, K. Z., et al. 2006, Acta Astron., 56, 333

Svensson, K. M., Ochoa-Lara, M. T., Lovey, F., et al. 2010, MNRAS, 405, 57

Tan, W. W., Cao, X. F., Yu, Y. W., 2013, ApJL, 772, L8

Tanvir, N. R., Barnard, V. E., Blain, A. W., et al. 2004, MNRAS, 352, 1073

Tanvir, N. R., Fox, D. B., Levan, A. J., et al. 2009, Nature, 461, 1254

Tegmark, M., Silk, J., Rees, M. J., et al. 1997, ApJ, 474, 1

Thompson, C., 2006, ApJ, 651, 333

Thöne, C. C., Fynbo, J. P. U., Goldoni, P., et al. 2013, MNRAS, 428, 3590

Thornton, D., Stappers, B., Bailes, M., et al. 2013, Science, 341, 53

Toma, K., Sakamoto, T., Mészáros, P. 2011, ApJ, 731, 127

Totani, T., 1997, ApJ, 486, L71

Totani, T., Kawai, N., Kosugi, G., et al. 2006, PASJ, 58, 485

Totani, T., Kentaro, A., Takashi, H., et al., 2014, PASJ, 66, 63

Trenti, M., et al., 2015, arXiv: 1406.1503

Tsutsui, R., et al., 2008, MNRAS, 394, L31

Tumlinson, J. 2010, ApJ, 708, 1398

Urata, Y. et al., 2009, ApJ, 706, L183

Valageas, P. 2000, A\&A, 354, 767

Vaughan, S. et al., 2006, ApJ, 638, 920

Vink, J. S., de Koter, A. 2005, A\&A, 442, 587

Virgili, F. J., Zhang, B., Nagamine, K., Choi, J. H. 2011, MNRAS, 417, 3025

Visser, M. 2004, Class. Quant. Grav., 21, 2603

Vitagliano, V., et al., 2010, JCAP, 03, 005

Wanderman, D., Piran, T., 2010, MNRAS, 406, 1944

Wang, F. Y., 2012, A\&A, 543, A91

Wang, F. Y., 2013, A\&A, 556, A90

Wang, F. Y., Bromm, V., Greif. T. H., et al. 2012, ApJ, 760, 27

Wang, F. Y., Dai, Z. G. 2006a, MNRAS, 368, 371

Wang, F. Y., Dai, Z. G. 2006b, Chin. J. Astron. Astrophys., 6, 561

Wang, F. Y., Dai, Z. G. 2009, MNRAS, 400, L10

Wang, F. Y., Dai, Z. G. 2011a, ApJL, 727, 34

Wang, F. Y., Dai, Z. G. 2011b, A\&A, 536, A96

Wang, F. Y., Dai, Z. G. 2014a, ApJS, 213, 15

Wang, F. Y., Dai, Z. G. 2014b, Phys. Rev. D, 89, 023004

Wang, F. Y., Dai, Z. G. 2013, Nature Physics, 9, 465

Wang, F. Y., Dai, Z. G., Qi, S. 2009a, A\&A, 507, 53

Wang, F. Y., Dai, Z. G., Qi, S. 2009b, RAA, 9, 547

Wang, F. Y., Dai Z. G., Zhu Z. H., 2007, ApJ, 667, 1

Wang, F. Y., Qi, S., Dai, Z. G. 2011, MNRAS, 415, 3423

Wang, F. Y., Yi, S. X., Dai, Z. G. 2014, ApJL, 786, L8

Wang, J. S., Wang, F. Y. 2014, A\&A, 564, A137

Wang, J. S., Wang, F. Y. 2014, MNRAS, 443, 1680 
Wang, Y., Fan, Y. Z., Wei, D. M., 2011, Phys. Rev. Lett., 106, 259001

Wang, Y., 2008, Phys. Rev. D, 78, 123532

Wang, Y., Mukherjee, P. 2006, ApJ, 650, 1

Waxman, E., Kulkarni, S. R., Frail, D. A., 1998, ApJ, 497, 288

Wei, D. M., Gao, W. H., 2003, MNRAS, 345, 743

Wei, H., 2010, JCAP, 008, 020

Wei, J. J., Wu, X. F., Melia, F., 2013, ApJ, 772, 43

Wei, J. J., Wu, X. F., Melia, F., et al., 2014, MNRAS, 439, 3329

Wijers, R. A. M. J. et al., 1998, MNRAS, 294, L13

Willingale, R. et al., 2007, ApJ, 662, 1093

Wolf, C., Podsiadlowski, P., 2007, MNRAS, 375, 1049

Woosley, S. E. 1993, ApJ, 405, 273

Woosley, S. E., Bloom, J. S., 2006, ARA\&A, 44, 507

Woosley, S. E., Heger, A. 2006, ApJ, 637, 914

Woosley, S. E., Weaver, T. A. 1995, ApJS, 101, 181

Wu, S. W., Xu, D., Zhang, F. W., Wei, D. M., 2012, MNRAS, 423, 2627

Wyithe, J. S. B., Loeb, A. 2003, ApJ, 586, 693

Xia, J. Q., 2012, Phys. Rev. D, 85, 043520

Xu, C. Y., Wei, D. M. 2008, Acta Astron. Sin., 49, 387

Xu, D., Dai, Z. G., Liang. E. W. 2005, ApJ, 633, 603

Xu, M., Huang, Y. F., 2012, A\&A, 538, A34

Yamazaki, R., Ioka, K., Nakamura, T., 2004, ApJ, 606, L33

Yonetoku, D. et al., 2004, ApJ, 609, 935

Yoon, S. C., Dierks, A., Langer, N., 2012, A\&A, 542, A113

Yoon, S. C., Langer, L. 2005, A\&A, 443, 643

Yoon, S. C., Langer, L., Norman, C. 2006, A\&A, 460, 199

Yoshida, N., Abel, T., Hernquist, L., Sugiyama, N. 2003, ApJ, 592, 645

Yu, B., Qi, S., Lu, T., 2009, ApJ, 705, L15

Yu, Y. W., Cheng, K. S., Chu, M. C., et al. 2012, JCAP, 07, 023

Yüksel, H., Kistler, M. D., 2007, Phys. Rev. D, 75, 083004

Yüksel, H., Kistler, M. D., Beacom, J. F., Hopkins, A. M., 2008, ApJ, 683, L5

Zhang, B., 2006, Nature, 444, 1010

Zhang, B., 2007, Chin. J. Astron. Astrophys., 7, 1

Zhang, B., 2014, ApJ, 780, L21

Zhang, B., 2014, Int. J. Mod. Phys. D, 23, 1430002

Zhang, B., et al., 2009, ApJ, 703, 1696

Zhang, B., Mészáros, P., 2002, ApJ, 581, 1236

Zhao, G. B., et al., 2012, Phys. Rev. Lett., 109, 17130

Zhou, B., Li, X., Wang, T., Fan, Y. Z., Wei, D. M. 2014, Phys. Rev. D, 89, 107303 